Uta Quasthoff, Christian Kluger

\title{
Familiale Interaktionsmuster als Erwerbsressource im längsschnittlichen Verlauf
}

\author{
Wie nutzen Kinder interaktive Ressourcen zum Ausbau \\ mündlicher Argumentationskompetenz?
}

\begin{abstract}
Wir betrachten in diesem Kapitel argumentative Familieninteraktionen mit Kindern der Sekundarstufe 1 als Erwerbskontext für argumentative Fähigkeiten. Dabei fragen wir, ob und wie die längsschnittliche Beobachtung der Familien hinsichtlich der drei familialen Interaktionsmuster Fordern und Unterstützen, Raumlassen und Akzeptieren sowie Übergehen und Selberlösen über die Sekundarstufe 1 hinweg offenlegen kann, welche interaktiven Ressourcen das Kind jeweils für seinen Erwerb von Argumentationskompetenz nutzen kann bzw. tatsächlich nutzt. Wir fokussieren dabei auf die Anteile des Kindes an den Aufgaben der Gesprächsorganisation, der Entscheidungsfindung und der Argumentation i. e. S. über drei Erhebungszeitpunkte. So konnten wir systematische Veränderungen von Ausmaß und Art der kindlichen Gesprächsbeiträge in den drei Bereichen Gesprächsorganisation, Etablierung der konversationellen Jobs und Mittel des Begründens über die Zeit ermitteln. Anhand von ausgewählten typischen Transkripten von Eltern-Kind-Interaktionen mit den Interaktionsmustern Fordern und Unterstützen sowie Raumlassen und Akzeptieren mit Kindern von der 6. bis zur 9. Klasse führen wir die subtilen Wirkmechanismen der Erwerbssupportivität im längsschnittlichen Vergleich vor. Abschließend zeigen wir anhand von Häufigkeitsverteilungen die Erwerbswirksamkeit von Fordern und Unterstützen und betrachten das Vorkommen dieses Musters in Familien mit unterschiedlichem Sozialstatus.
\end{abstract}

Keywords: Argumentieren, Diskurskompetenz, Interaktionsmuster, Fordern und Unterstützen, Entscheidungsdiskurs, Familie, Erwerbsunterstützung, Erwerbsressource, Finetuning

Uta Quasthoff, TU Dortmund, Emil-Figge-Straße 50, 44227 Dortmund, uta.quasthoff@tu-dortmund.de

Christian Kluger, TU Dortmund, Martin-Schmeißer-Weg 13, 44221 Dortmund, christian.kluger@tu-dortmund.de

Ә Open Access. ( 2021 Uta Quasthoff, et al., publiziert von De Gruyter. (c))BY-NC-ND Dieses Werk ist lizensiert unter einer Creative Commons Namensnennung - Nicht-kommerziell - Keine Bearbeitung 4.0 International Lizenz. 


\section{Einführung und Fragestellung}

Die Art der Erwachsenen-Kind-Interaktion im Rahmen der alltäglichen familialen Kommunikationspraxis spielt eine zentrale Rolle für den Spracherwerb des Kindes. Diese Einsicht verdanken wir - grob gesprochen - mindestens zwei unterschiedlichen Forschungsfeldern: (1) Die linguistische und psychologische Spracherwerbsforschung beschäftigt sich spätestens seit den 70er Jahren des vorigen Jahrhunderts und insbes. seit Bruners Konzept eines Language Acquisition Support System (LASS: Bruner 1978) mit der Frage, in welcher Weise der Spracherwerb des Kindes - zusätzlich zu angeborenen Dispositionen - auf bestimmte Strukturen seiner sprachlichen Umgebung zurückgreift. (2) Die interdisziplinäre Bildungsforschung nimmt, insbesondere seitdem die großen internationalen Vergleichsstudien immer wieder eine Beeinträchtigung des Schulerfolgs durch sprachliche Benachteiligung feststellten (Stanat \& Edele 2014), die sprachsozialisatorischen Bedingungen im Elternhaus (Tillmann 2001) und später auch im Unterricht (Becker-Mrotzek et al. (Hrsg.) 2013) in den Blick. Dabei fokussierte die Spracherwerbsforschung eher auf das regelmäßige Zusammenspiel zwischen den phonologischen, grammatischen und lexikalischen Strukturen des sprachlichen ,Input' des Erwachsenen (Snow \& Ferguson (Hrsg.) 1977) und später v. a. auch auf die dialogischen Merkmale von child directed speech (Snow 1995) als Bedingung von Spracherwerbsprozessen generell. Die Bildungsforschung interessierte sich dagegen eher für die Unterschiede in den sprachlichen Sozialisationsbedingungen verschiedener Kinder mit dem Ziel, die vorfindlichen Kompetenzunterschiede bei der Sprachbeherrschung (Rowe 2008) und nachfolgend im schulischen Lernen erklären zu können.

Die Forschung, die unserem Beitrag i.e.S. zugrunde liegt, verbindet in einem interdisziplinär linguistisch-psychologischen Zugriff diese beiden Forschungstraditionen (Krah et al. 2013; Quasthoff \& Wild 2014; Quasthoff, Wild et al. 2015; Quasthoff, Wild et al. in diesem Band). In den Studien FUnDuS I und II $^{1}$ wurde gefragt, in welcher Abfolge und in welcher Weise Kinder im Verlauf der Sekundarstufe I in familialer Interaktion eine besonders (sekundar-) schulrelevante Ausprägung der Diskurskompetenz (vgl. zu diesem und anderen grundlegenden Konzepten das Glossar in diesem Band) erwerben, nämlich Argumentationskompetenz. Dabei interessierte uns besonders, ob und in welcher

1 Die Rolle familialer Unterstützung beim Erwerb von Diskurs- und Schreibfähigkeiten in der Sekundarstufe I (FUnDuS); Ltg. Uta Quasthoff und Elke Wild, gefördert vom BMBF, Förderkennzeichen 01 GJ 1207/A + B. 
Weise die Familie als Erwerbskontext den Erwerb der Argumentationskompetenz beeinflusst. Den Theoriehintergrund bildete in linguistischer Perspektivierung die Spracherwerbs- und die soziolinguistische Sozialisationsforschung (Burleson, Jesse \& Applegate 1995; Steinig 2016) sowie die speziellen langjährigen Forschungserfahrungen zu den interaktiven Mechanismen des Diskurserwerbs im Rahmen des Discourse Acquisition Support System (DASS) (Hausendorf \& Quasthoff 1996, 2005; Kern 2011; Morek, Heller \& Quasthoff 2017).

Empirisch wurden in FUnDuS längsschnittlich zum einen die Erwerbsabfolgen von der 6. bis zur 9. Klasse quantitativ in einer großen (Ausgangs-)Stichprobe von mehr als 1400 Schülerinnen und Schülern ermittelt (vgl. Quasthoff, Wild et al. in diesem Band). Zum anderen wurden im Rahmen einer deutlich kleineren Intensivstichprobe ( $\mathrm{N}=33$ im Längsschnitt) die Unterschiedlichkeit familialer Interaktionsformen sowie ihre Veränderung über den Zeitraum der Sekundarstufe I kontextuell rekonstruiert. Ziel dieser qualitativen Analyse war u. a. einen Zusammenhang zwischen familialen Interaktionsmustern und kindlichen Kompetenzniveaus bzw. Erwerbsverläufen zu überprüfen.

Im vorliegenden Beitrag geht es weniger um den Erwerbsverlauf, sondern um den Einfluss des Erwerbskontextes: Wir fragen, ob und wie die längsschnittliche Beobachtung der Interaktionsmuster von Eltern-Kind-Dyaden offenlegen kann, welche unterschiedlichen ,externen', interaktiven Ressourcen (Quasthoff 2011; Quasthoff et al. 2019; Quasthoff, Heller \& Morek in diesem Band) das Kind jeweils für seinen Erwerb von Argumentationskompetenz nutzen kann bzw. tatsächlich nutzt und wie diese unterschiedlichen Ressourcen mit der Kompetenzentwicklung zusammenhängen.

Das zentrale Konstrukt der mündlichen und schriftlichen Argumentationskompetenz (Willenberg, Gailberger \& Krelle 2007; Feilke 2013; Arendt 2019) fassen wir - dem Theorierahmen der Interaktionalen Diskursanalyse (Quasthoff, Heller \& Morek 2017; Morek, Heller \& Quasthoff 2017) folgend - im Mündlichen als eine gattungsspezifische (Bergmann \& Luckmann 1995) Art von Diskursfähigkeit (Quasthoff \& Katz-Bernstein 2006). Das Konzept Diskursfähigkeit bezeichnet das Vermögen, über Grammatik und Lexik hinaus solche sprachlichen Praktiken angemessen vollziehen und kontextuell platzieren zu können, die typischerweise äußerungsübergreifend, also diskursiv, organisiert sind und die sich erkennbar an den Merkmalen einer diskursiven Gattung wie etwa dem Erzählen, Erklären oder eben auch dem Argumentieren orientieren.

Wie Diskurskompetenz generell (Quasthoff et al. 2019) gliedert sich auch die Argumentationskompetenz (im Folgenden: AK) wesentlich in drei sprachliche Facetten (Vertextung: interner Aufbau, Kontextualisierung: kontextuelle Einbettung sowie erkennbare sprachliche Markierung, Quasthoff, 2009; Quasthoff \& Dome- 
nech 2016; Quasthoff et al. 2019; Quasthoff, Heller \& Morek in diesem Band). Das Konstrukt von AK, das wir in FUnDuS zugrunde gelegt und sowohl mündlich wie schriftlich operationalisiert haben und empirisch validieren konnten (Quasthoff \& Domenech 2016; Quasthoff, Wild et al. in diesem Band) setzt sich darüber hinaus aus rezeptiven und produktiven Anteilen zusammen und ist an kognitive Voraussetzungen (z. B. Fähigkeit zu schlussfolgernden Denken) gebunden. In einer Begrifflichkeit von Ehlich (2012) kann Argumentieren persuasive und explorative Funktionen erfüllen, die beide in der FUnDuS-Studie untersucht wurden.

Vor diesem Hintergrund hat der folgende Beitrag das Ziel nachzuzeichnen, wie sich die musterhaft unterschiedlichen argumentativen Interaktionen in Eltern-Kind-Dyaden mit Sechstklässlern verändern, wenn das Kind älter und kompetenter wird. An diesen Prozessen untersuchen wir,

- in welchen Gesprächsbereichen sich Veränderungen in der Rolle und Kompetenz des Kindes ggf. zeigen;

- ob und wie sich das elterliche Gesprächsverhalten an die Kompetenz des älterwerdenden Kindes anpasst (Finetuning, Snow 1995; Morek \& Heller in diesem Band);

- ob und wie die unterschiedlichen familialen Interaktionspraktiken dem Kind jeweils Erwerbsressourcen bieten und ob und wie diese von den Kindern genutzt werden.

Auf dieses Weise werden wir die besondere Erwerbssupportivität des Musters Fordern und Unterstützen vorführen (Quasthoff \& Kern 2007; Heller \& Krah 2015; Quasthoff, Heller \& Morek in diesem Band; Domenech \& Krah in diesem Band; Morek \& Heller in diesem Band) und in seiner Wirksamkeit exemplarisch offenlegen, gerade auch anhand eher subtiler Unterschiede zu einem anderen Interaktionsmuster.

Wir berichten im folgenden Abschnitt 2 kurz über den einschlägigen Forschungskontext, beschreiben im Abschnitt 3 die Daten und unsere analytische Herangehensweise in der FUnDuS-Studie, bevor wir im Abschnitt 4 das genaue Vorgehen für die vorliegende Längsschnitt-Analyse beschreiben. Anhand von ausgewählten typischen Transkripten von Eltern-Kind-Interaktionen mit ähnlichen Interaktionsmustern führen wir im Abschnitt 5 die subtilen Wirkmechanismen vor. Im Abschnitt 6 schließlich diskutieren wir abschließend die Erwerbswirksamkeit von Fordern und Unterstützen und betrachten die Häufigkeit des Vorkommens in Familien mit unterschiedlichem Sozialstatus. 


\section{Bisherige Forschungen zur Funktion der Erwachsenen-Kind-Interaktion beim Erwerb von Diskursfähigkeiten}

Unter den linguistischen Beschreibungsebenen bezeichnet die Diskurs- bzw. Textebene Merkmale und Verfahren regelhafter Strukturierung oberhalb der Ebene einzelner Äußerungen bzw. Sätze. Einschlägige Arbeiten zum Diskurserwerb (Hausendorf \& Quasthoff 2005) betrachten folglich die zunehmende Kompetenz der Kinder, in Gesprächen erkennbare ,Diskurseinheiten“ (Wald 1978; Quasthoff, Heller \& Morek 2017) bzw. ,Handlungsmuster‘ (Ehlich \& Rehbein 1986) wie Erzählungen, Wegbeschreibungen, Erklärungen, Instruktionen, Argumente intern aufzubauen und sprachlich zu gestalten. Die Interaktionale Diskursanalyse betrachtet zusätzlich systematisch die Fähigkeit, Diskurseinheiten in den Gesprächskontext zu platzieren, und bezieht die Vertextung und sprachlich-formale Gestaltung auf die jeweils gattungsspezifischen Äußerungspakete als Teil der Diskurseinheit. Empirische Arbeiten zur Entwicklung von Erklärungen (Klann-Delius et al. 1985; Hausendorf 1995) oder Argumenten (Völzing 1981; Miller 1980; Goetz 2010) finden sich gegenüber der dominanten Forschung zum Erzählerwerb immer noch eher selten (vgl. den Überblick in Morek, Heller \& Quasthoff 2017). Allerdings erfahren diese beiden Diskursgattungen, sicher nicht zuletzt auf Grund ihrer kognitiven und schulischen Relevanz, gegenwärtig erhöhte Aufmerksamkeit (z. B. Spreckels 2008; Arendt, Heller \& Krah (Hrsg.) 2015; Heller \& Morek 2015; Morek, Heller \& Quasthoff 2017; Arendt 2019), wobei das Argumentieren häufig ausschließlich im schulischen Kontext (Erath \& Prediger 2014; Grundler 2011; Spiegel 2006; Krelle 2014) betrachtet und oft mit Fokus auf der Schriftlichkeit (Feilke 2013; Rezat 2011) untersucht wird.

Mündliche argumentative Diskurse lassen sich unterscheiden in solche, die inkompatible, strittige Positionen bearbeiten - oft mit dem kommunikativen Ziel der wechselseitigen Überzeugung (persuasives Argumentieren), und solche, die eine offene Frage (,Quaestio') mit Hilfe von Argumenten (exploratives Argumentieren, Ehlich 2012) bearbeiten. Letztere ähneln also eher einem Problemlösediskurs mit argumentativen Mitteln, der nicht nur am Kriterium der Gültigkeit, sondern auch an Gesichtspunkten wie Zweckmäßigkeit oder Angemessenheit orientiert ist. Argumentative Diskurse stellen besondere strukturelle Anforderungen an die beteiligten Interaktanten, die sich $\mathfrak{u}$. a. aus ihrer komplexen sequenziellen Konstellationen zwischen strittigen Positionen bzw. offenen Fragen, stützenden Argumenten und verwendeten Schlussregeln ergeben. Auch die prinzipiell gleichberechtigten Rollen von Proponent und Opponent erzeugen 
einen erhöhten Organisationsbedarf (Quasthoff, Heller \& Morek 2017). Insgesamt zeigen vorliegende Studien zum Erwerb von Argumentationskompetenz älterer Kinder (zsf. Morek, Heller \& Quasthoff 2017; Heller \& Krah 2015, Quasthoff \& Domenech 2016), dass die Qualität des (mündlichen) Argumentierens im Verlauf der Schullaufbahn eine große Varianz aufweist (Spiegel 2006; Grundler 2011; Krelle 2014).

Arbeiten, die die Funktionalität alltäglicher informeller dialogischer Unterstützungssysteme der Erwachsenen-Kind-Interaktion (Hausendorf und Quasthoff 1996) für den Aufbau globaler mündlicher Diskursfähigkeiten beleuchten (s. auch Quasthoff in diesem Band), zielen insbesondere auf die Erklärung produktiver (mündlicher) Diskurserwerbsprozesse der Kinder ab. Die rekonstruktiven Analysen zeigen die unmittelbar interaktive und im Altersvergleich sichtbare ontogenetische Wirksamkeit des Disourse Acquisition Support System (DASS, Hausendorf \& Quasthoff 1996). In mikroanalytischer Rekonstruktion dieses dialogischen Prinzips der Erwachsenen-Kind-Interaktion, das in unterschiedlichen querschnittlich betrachteten Altersgruppen jeweils angepasst funktionierte, gelang es, die erwerbssupportive Wirkweise dieser Interaktionspraktik mit Bezug auf die kindlichen Erzählfähigkeiten herauszuarbeiten (Hausendorf \& Quasthoff 2005). Theoretisch werden Muster der Erwachsenen-Kind-Interaktion als spezifische Kontextualisierung allgemeiner Interaktionsprinzipien wie recipient design (Sacks 1995) erkannt. Dieser Gesichtspunkt weist darauf hin, dass diese Art des Discourse Acquisition Support System in Alltagsgesprächen kommunikativ und nicht didaktisch motiviert ist: Es funktioniert, weil Erwachsene ihre kindlichen Gesprächspartner verstehen und nicht, weil sie in einer Art von Lehr-Lern-Diskurs den Kindern angemessenes Sprachverhalten vermitteln wollen (Krah 2017).

Breitere empirische Untersuchungen zur Verbreitung erwerbsförderlicher Erwachsenen-Kind-Interaktionen (Quasthoff \& Kern 2007; Morek 2012; Heller 2012) zeigten, dass der sprachlich-interaktive Umgang von Eltern mit kindlichen Diskursen unterschiedlich ausfällt (Heller \& Krah 2015). Als erwerbsförderlich erwiesen sich in theoretischer Übereinstimmung mit Hausendorf \& Quasthoff (1996) solche Interaktionsmuster, in denen den Kindern ein vergleichsweise großer gesprächsstruktureller Raum zugebilligt wurde, der verständigungsorientiert durch Zuhöreraktivitäten unterstützend mitgestaltet wurde. Diese Praktik der Erwachsenen-Kind-Interaktion wurde als Muster Fordern und Unterstützen beschrieben (Quasthoff \& Kern 2007; s. u. Abschnitt 3). Die entsprechende spracherwerbstheoretisch beschriebene familiale Kommunikationspraktik wurde in FUnDuS theoretisch verbunden mit den Erkenntnissen zu den positiven Effekten einer autonomieunterstützenden Erziehung und Hilfe beim häuslichen Lernen (Walper \& Wild 2014) sowie dem ,sprachlichen Anregungsgehalt' (Hollmann et al. 2012; 
Quasthoff, Wild et al. in diesem Band). Auf diese Weise konnte die Wirksamkeit von Fordern und Unterstützen mittelbar auch quantitativ bestätigt werden.

Der vorliegende Beitrag kann sich - auch in der Aufarbeitung des einschlägigen Forschungsstands - sehr weitgehend auf den Artikel von Heller \& Krah 2015 stützen, der dieselben Daten aus FUnDuS unter ähnlichen Fragestellungen bearbeitet (s. auch Quasthoff et al. 2015). Die genaue Rekonstruktion der längsschnittlichen Veränderungen in den Interaktionsformen der Erwachsenen-Kind-Dyaden konnte dort zeigen, auf welche Weise Fordern und Unterstützen im Unterschied zu anderen familialen Praktiken erwerbsunterstützend wirkt. Im Unterschied zur genannten Arbeit fokussieren wir hier auf die kindlichen Anteile an den Aufgaben der Gesprächsorganisation, der Entscheidungsfindung und der Argumentation über alle drei Erhebungszeitpunkte und vergleichen dabei Fordern und Unterstützen mit einem sehr ähnlichen Muster.

\section{Daten und analytisches Vorgehen in FUnDuS}

\subsection{Daten}

Im Rahmen des Projektdesigns von FUnDuS sollten die qualitativen Analysen zur Varianz des erwerbsförderlichen Interaktionsverhaltens von Eltern-KindDyaden mehrere Fragen beantworten:

(1) Behalten die Familien ihre familialen, jeweils mehr oder weniger erwerbsförderlichen Interaktionsmuster über die Zeit des Längsschnitts bei?

(2) Lässt sich im Längsschnitt die erwerbsförderliche Mechanik nachzeichnen?

(3) Lässt sich dabei eine Anpassung an das sich verändernde Alter der Kinder beobachten?

(4) Sind die familialen Kommunikationskulturen über die Zeit an den jeweiligen sozioökonomischen Status gebunden (s. auch Quasthoff, Wild et al. in diesem Band; Quasthoff in diesem Band)?

Im Fall einer engen Kopplung zwischen sozioökonomischem Status und Argumentationsverhalten wären die Praktiken schlecht durch ein Elterntraining beeinflussbar gewesen - genau das war jedoch in FUnDuS geplant (Kluger in diesem Band).

Auf der Grundlage der großen Ausgangsstichprobe von über 1400 Schülerinnen und Schülern aus Hauptschulen und Gymnasien wurde deshalb systematisch eine Teilstichprobe gezogen, die sich aus drei Gruppen zusammensetzte: (1) sozial privilegierte Kinder mit vergleichsweise wenig entwickelten argumentativen Kom- 
petenzen (,social underachiever'), (2) Gleichaltrige aus bildungsfernen Elternhäusern, deren Argumentationskompetenz überdurchschnittlich gut entwickelt ist (,social overachiever'), und (3) die ,erwartungskonformen' Schülerinnen und Schüler, also die Kinder, die eine gemäß ihrer sozialen Herkunft erwartbar hoch bzw. niedrig entwickelte Kompetenz aufweisen (zu den Verfahren bei der Stichprobenziehung s. genauer Krah et al. 2013; zum Zusammenhang von Stichprobe, Korpus und Fragestellung s. Quasthoff in diesem Band).

Die Erhebung in der Intensivstichprobe beabsichtigte außerdem - ebenfalls im Hinblick auf die Entwicklung eines Trainings - diejenigen interaktiven Verfahren von Eltern und Kindern zu elizitieren, die diese anwenden, wenn die Eltern es in klar aufgabenfokussierter Interaktion aus ihrer Sicht möglichst „gut machen“ wollen. Es ging also ausdrücklich nicht um die Erhebung ,natürlicher` Alltagsroutinen (Quasthoff in diesem Band). Entsprechend wurden nach sprachdidaktischen Kriterien drei Aufgabenformate konzipiert und pilotiert, die einerseits ein hinreichend motivierendes Anreizpotential für die Beteiligten aufweisen und andererseits Raum lassen für die Realisierung unterschiedlicher Interaktionsmuster der Dyaden mit verschiedenen Beteiligungsmöglichkeiten für die Kinder. Im Längsschnitt mit drei Erhebungszeitpunkten (im Folgenden EZPe) in der 6., 7. und 9. Jahrgangsstufe wurden die Aufgabenformate strukturell beibehalten und lediglich inhaltlich leicht verändert.

Diese Aufgaben umfassen (1.) eine argumentative Schreibaufgabe für das Kind. Die entstehenden Kindertexte dienen (2.) als Grundlage für eine kooperative Überarbeitungsaufgabe, in der Elternteil und Kind gebeten werden, gemeinsam den Text „noch besser zu machen“. Als rein mündliche Aufgabe wurden (3.) Kind und Elternteil gebeten, im Gespräch gemeinsam zu begründeten Entscheidungen zwischen jeweils vier familienrelevanten Optionen zu kommen. Hierbei handelt es sich um eine konsensuale explorative Argumentationsaufgabe (,einigt Euch und begründet“). Die Aufzeichnungen zu dieser Aufgabe liegen den nachfolgenden Analysen wesentlich zugrunde.

Alle Eltern-Kind-Interaktionen fanden im häuslichen Milieu der Familien statt. Sie wurden videographiert und nach dem Gesprächsanalytischen Transkriptionssystem (GAT 2, Selting et al. 2009) unter Nutzung des Tools Transana transkribiert (s. die wichtigsten Notationen von GAT im Anhang dieses Bandes). Um die Vergleichbarkeit der Durchführungen sicherzustellen, wurden alle Untersuchungsleiter/innen anhand eines ausführlichen Leitfadens intensiv geschult. Nach Pilotierung haben sich die eingesetzten Aufgaben als außerordentlich fruchtbar erwiesen. Das gilt sowohl im Hinblick auf die Durchführbarkeit bei den Familien zuhause als auch mit Blick auf die angestrebte Erfassung einer großen Bandbreite unterschiedlicher Praktiken im Umgang mit mündlichen und textbezogenen Aufgabenstellungen im Verlauf der Altersentwicklung. 


\subsection{Analyseverfahren und -ergebnisse der qualitativen FUnDuS-Studie}

Die Analyse dieser Daten von längsschnittlich erfassten Eltern-Kind-Interaktionen über drei EZPe hinweg orientierte sich an konversationsanalytischen Verfahren der Sequenzanalyse und nutzte das Analyseverfahren GLOBE (Hausendorf \& Quasthoff 2005; Quasthoff, Heller \& Morek 2017; speziell zu den FUnDuS-Daten: Heller \& Krah 2015). Die Analyse der im Rahmen von GLOBE sequenziell zu rekonstruierenden, von den Beteiligten gemeinsam zu erledigenden argumentativen Jobs konnte dabei auf die in Heller (2012) explizierten Jobs zurückgreifen:

- Dissens herstellen/Problematisieren

- Begründungspflicht etablieren

- Kernaufgabe Begründen

- Abschließen

- Überleiten

Die mikroprozessuelle Analyse der FUnDuS-Studie legte offen, dass die Beteiligten in ihren Gesprächen zur mündlichen Entscheidungsaufgabe sich wechselseitig jeweils anzeigen, ob sie im Aufgaben-, Entscheidungs- oder Argumentationsdiskurs agieren. Sie machen also jeweils erkennbar, ob sie gerade die von uns gestellte Interaktionsaufgabe ${ }^{2}$, die Auswahl zwischen den vier gegebenen Entscheidungsoptionen $^{3}$ oder die begründende Argumentation für oder gegen eine jeweilige Option relevant setzen (s. auch Quasthoff in diesem Band).

Die strikt induktive Analyse der qualitativen FUnDuS-Daten ergab weiterhin, dass die Varianz der Praktiken in den Eltern-Kind-Dyaden erschöpfend in drei Interaktionsmustern gefasst werden konnte, die sich als kontextuell bedingte Varianten bereits zuvor beschriebener (Quasthoff \& Kern 2007; Morek 2012; Heller 2012; s. o. Abschnitt 2) Eltern-Kind-Interaktionsmuster erwiesen. Die aus den FUnDuS-Daten herauspräparierten Interaktionsmuster Forderm und Unterstützen, Raumlassen und Akzeptieren sowie Übergehen und Selberlösen (Krah et al. 2013; Quasthoff et al. 2015; Quasthoff \& Krah 2012; Heller \& Krah 2015) charakterisieren die speziellen Praktiken, die die beteiligten Eltern in ihrer Steuerungsrolle in den ineinander verschachtelten Aufgaben-, Entscheidungs- und Argumentationsdiskursen vollzogen.

Entsprechend der ethnomethodologischen Grundorientierung wurden bisher gefundene Muster nicht als Erwartungen an die Daten herangetragen, sondern induktiv als musterhaft kondensierte Praktiken der Dyaden rekonstruiert.

2 bezogen auf die Instruktion, die durch ein Mitglied des Forscherteams verlesen wurde.

3 lagen den Beteiligten als Handout vor. 
Hintergrund dieser Sequenzanalyse ist die Mechanik von Zugzwängen in jeglicher Interaktion, die von der klassischen Konversationsanalyse zunächst primär im Zusammenhang mit adjacency pairs wie Frage-Antwort untersucht wurde (Schegloff 1972). Die Interaktionale Diskursanalyse hat das Konzept derartiger lokaler Zugzwänge erweitert um das Konzept globaler Zugzwänge, die die sequenzielle Verpflichtung zum Anschluss einer globalen Einheit wie z. B. einer Erklärung oder eines Arguments etablieren (Hausendorf \& Quasthoff 1996; Quasthoff, Heller \& Morek 2017).

Die Muster lassen sich - fokussiert auf den Argumentationsdiskurs - jeweils durch die folgenden Merkmale charakterisieren (Krah et al. 2013; Heller \& Krah 2015):

\section{Fordern und Unterstützen}

Das Elternteil

- lässt kontextuell angemessen Gesprächsraum für kindliche Gesprächsbeiträge;

- unterstützt das Kind durch Setzen von lokalen bzw. globalen Zugzwängen (Hausendorf \& Quasthoff 1996; Quasthoff, Heller \& Morek 2017) je nach unmittelbarem Bedarf des Kindes (Finetuning);

- lässt jede Position des Kindes zu, auch wenn sie von der eigenen abweicht;

- fordert das Kind im Falle noch unklarer inhaltlicher Aussagen zu weiteren Elaborierungen / Begründungen auf;

- begründet auch die eigene Position.

Grundsätzlich wird im Rahmen dieses Musters, also im Dialog, gefordert, indem das Kind durch entsprechende Züge des Erwachsenen unter die sequenzielle Verpflichtung gestellt wird, möglichst umfassend und elaboriert $\mathrm{zu}$ begründen und abzuwägen. Gleichzeitig wird das Kind bei dieser sequenziell gesetzten Aufgabe durch dialogische Hilfen (lokale Nachfragen, eigene Erwägungen etc.) unterstützt. Dies geschieht kontextbezogen im Rahmen der Adressatenorientierung jeglicher Interaktion in dem Maße, in dem das Kind wirklich Unterstützungsbedarf zeigt: Das Finetuning reagiert also intuitiv und implizit auf das Kompetenzniveau des Kindes und sollte entsprechend dem zunehmendem Alter der Kinder im Längsschnitt zu angepassten Verfahren führen (s. u. Abschnitte 4 und 5).

\section{Raumlassen und Akzeptieren}

Das Elternteil

- bietet (wie bei Fordern und Unterstützen) den notwendigen Gesprächsraum für kindliche Äußerungen; 
- formuliert Nachfragen oder Einwände allenfalls global oder produziert sie gar nicht;

- steuert selbst eher ergebnisorientierte (statt begründend-abwägende) Verfahren der Entscheidungsfindung bei;

- akzeptiert die Beiträge des Kindes, führt sie aber selten im weiteren Diskurs fort;

- passt sich entsprechend nicht Schritt-für-Schritt an den kindlichen Unterstützungsbedarf an (Finetuning).

Hier wird also zwar Gesprächsraum gelassen, aber keine elterliche Unterstützung zu dessen Füllung angeboten. Kindliche Positionen werden ohne weitere Auseinandersetzung akzeptiert, so dass häufig der Eindruck von nur lose verknüpften Interaktionssequenzen entsteht. Das Kind wird so im Dialog weder gefordert noch unterstützt, sondern eher allein gelassen.

\section{Übergehen und Selberlösen}

Das Elternteil

- übernimmt alle wesentlichen Aspekte der Entscheidungsfindung und Begründung selbst;

- duldet zumeist keine Position des Kindes, die von der eigenen abweicht, d. h. es übergeht diese oder lehnt sie unbegründet ab;

- fordert keine Begründungen ein;

- produziert auch selbst keine Einwände, da das Kind keinen Raum für eigene Begründungen erhält.

In diesem Muster wird das Kind also ebenfalls weder gefordert noch unterstützt. Im Unterschied zu Raumlassen und Akzeptieren lässt das Elternteil dem Kind darüber hinaus generell kaum Möglichkeiten zur Gesprächsbeteiligung und übergeht (z. T. durch Unterbrechungen) entsprechende Versuche des Kindes. Das Elternteil setzt die von ihm erarbeitete Lösung der Aufgabe und nicht die argumentative Verständigung mit dem Kind relevant, d. h. es löst die Aufgabe selbst. 


\section{Herangehensweise bei den längsschnittlichen Beobachtungen}

Angesichts der in Heller \& Krah (2015) bereits vorgestellten Interaktionsmechanik der drei Muster fokussiert die hier vorzustellende Längsschnittanalyse vor allem auf die kindlichen Beiträge. Wir betrachten, inwiefern ein Anstieg kindlicher Anteile im Diskurs zu verzeichnen ist und ob sich die kindlichen Aktivitäten unter den sequenziellen Bedingungen der jeweiligen Interaktionsmuster qualitativ verändern.

Die Übernahme größerer Anteile durch das Kind an der ko-konstruierten Erwachsenen-Kind-Interaktion sowie die Art der vom Kind beigesteuerten Diskursaktivitäten, ohne dass ihnen eine lokale Erwachsenen-Unterstützung vorausgegangen wäre (s. u.), wurden im Rahmen der Erwerbstheorie der Interaktionalen Diskursanalyse als Indikatoren für Erwerbsprozesse herausgearbeitet (Hausendorf \& Quasthoff 2005; Bergmann \& Quasthoff 2010; Quasthoff 2012). Nimmt man nämlich die Grundannahme der Konversationsanalyse und anderer interaktionaler Ansätze ernst, dass jegliche Kommunikation eine gemeinsam hervorgebrachte Leistung der Beteiligten ist, dann muss die kindliche ,Eigenleistung“ im Rahmen der vom Erwachsenen sequenziell unterstützten Kommunikation mit einigem methodischen Aufwand herauspräpariert werden. Die folgenden Transkriptanalysen (Abschnitt 5) werden im Einzelnen vorführen, dass und warum nach GLOBE nur selbstinitiierte kindliche Beiträge unter den kontextuellen Bedingungen der Steuerung und Ermöglichung durch die Zugzwänge des Elternteils analytisch dem Kind allein - und damit seiner Kompetenz - zugeschrieben werden können.

In einem ersten Schritt wurden alle Eltern-Kind-Dyaden pro EZP $(\mathrm{N}=33)$ in ihren musterhaften Praktiken verglichen. Auf der Grundlage dieses Überblicks über das gesamte Korpus erfolgte im zweiten Schritt die Rekonstruktion von Veränderungen im Längsschnitt besonders im Hinblick auf größere und andersartige argumentative Anteile des Kindes mit dem Ziel der Feststellung eines Kompetenzzuwachses.

Dies stellte sich angesichts der Spezifik der jeweils aktualisierten Interaktionspraktiken durchaus als methodische Herausforderung an die Kontextualität der Analyse der vergleichsweise großen Stichprobe heraus: Die charakteristischen Merkmale der unterschiedlichen Interaktionsmuster mussten in ihrer sequenziellen Einbindung und v. a. in ihrer jeweiligen mikrogenetischen Wirkung auf die kindlichen Anteile berücksichtigt werden. So musste nicht nur rekonstruiert werden, ob die jeweilige global relevante Äußerung des Kindes selbstinitiiert oder durch eine Vorgängeräußerung des Erwachsenen ausgelöst wurde, ob also der jeweilige Redezug als 
Indikator der eigenen globalen Leistung des Kindes gewertet werden konnte oder lediglich lokalen Antwortcharakter hat. Es musste auch erfasst werden, ob dem Kind überhaupt die Möglichkeit etwa zu begründenden Zügen gelassen wurde. So wäre es beispielsweise nicht sinnvoll gewesen, die Kompetenzentwicklung des Kindes allein anhand des Ausmaßes kindlicher Begründungen festzulegen, da das Muster Fordern und Unterstützen diese explizit einfordert und somit die reine Anzahl an (fremdinitiierten) Begründungen mikrogenetisch bedingt (Quasthoff 2012) höher ist als bei den anderen Interaktionsmustern. Übergehen und Selberlösen hingegen lässt kaum Raum für kindliche Begründungen, sodass hier die kontextuellen Gelegenheiten für das Vorkommen von Begründungen stark eingeschränkt sind.

Als wichtigster Indikator für die individuelle Entwicklung der Diskurskompetenz im Allgemeinen und der Argumentationskompetenz im Speziellen kristallisierte sich entsprechend die Selbstständigkeit (Quasthoff et al. 2011; Quasthoff et al. 2019) des Kindes bei der Produktion zunehmend anspruchsvoller Anteile bei Entscheidungs- und Argumentationssequenzen heraus. Es wurde also zum einen rekonstruiert, ob das Kind im längsschnittlichen Verlauf unter den lokalen kontextuellen Bedingungen der jeweiligen Muster im Verlauf selbstständiger erkannte, dass und wann es Begründungen von sich aus zu produzieren hat, ohne dass das Elternteil seinerseits durch einen entsprechenden Zugzwang (etwa durch eine Warum-Frage) die Begründungspflicht etablierte (Heller 2012). Dieser Analysestrang betrachtete also einen Aspekt der Kontextualisierungskompetenz (s. o. Abschnitt 1). Zum anderen wurde jeweils beleuchtet, ob diese Argumente zunehmend globaler und ausgebauter (Quasthoff \& Domenech 2016) organisiert wurden. Hier ging es also um die Facette der Vertextungskompetenz.

Im Ergebnis der gesamten Analyse stellte sich heraus, dass systematische Veränderungen von Ausmaß und Art der kindlichen Gesprächsbeiträge im Kontext der jeweiligen Interaktionsmuster in drei Bereichen beschrieben werden können:

Gesprächsorganisation: Hierzu gehört zum einen die globale Diskursorganisation des gesamten Gesprächs zu der mündlichen Aufgabe (s. o. Abschnitt 3.1). So machen Beteiligte z. B. die Vorschläge, alle vier Entscheidungsoptionen nacheinander durchzugehen oder nach dem Ausschlussverfahren vorzugehen. Zum anderen werden eher lokale Organisationsverfahren im Entscheidungsdiskurs betrachtet, z. B. Adressierung von Alternativoptionen, Bilanzieren bzw. Festhalten von Zwischenergebnissen. Die Gesprächsorganisation gehört i.e.S. nicht zur Argumentationsanalyse. Es ist aber eine Eigenheit unserer Daten, dass Aufgaben-, Entscheidungs- und Argumentationsdiskurs (s. auch Abschnitt 3.1 und 5) eng ineinander verschachtelt sind und sich sequenziell wechselseitig bedingen. 
Unser Ansatz der kontextuellen Rekonstruktion und unser Modell von Argumentationskompetenz, das die Kontextualisierungskompetenz einschließt, verlangen daher die Betrachtung der je spezifischen Dynamik auch der Gesprächsorganisation.

Etablierung der konversationellen Jobs: In diesem Analyseschritt wurde insgesamt untersucht, ob Kind oder Elternteil die konversationellen Jobs Begründungspflicht etablieren, Kernjob Begründen sowie Abschließen (vgl. Heller 2012, s. o. Abschnitt 2) maßgeblich etabliert. Hier wurde also auf die gattungsspezifischen Aktivitäten i. e. S. und dabei insbesondere auf die Kontextualisierungskompetenz geschaut. Im Zusammenhang mit der erwähnten ,Eigenleistung‘, also der methodischen Voraussetzung für die Zuschreibung von Kompetenz an das Kind, galt es jeweils zu überprüfen, ob der Job vom Kind selbstständig initiiert wurde oder ob das Elternteil ihn per Zugzwang ausgelöst hatte. Ein typischer elterlicher Zugzwang zum Job Begründungspflicht etablieren stellt - wie erwähnt - eine entsprechende Nachfrage dar, z. B. „Und warum bist du gegen den Segeltörn?“. Mit einem derartigen Zugzwang wird in aller Regel der Übergang zum Kernjob Begründen vom Elternteil sequenziell erwartbar gemacht. Eine daran angeschlossene Begründung des Kindes (,weil man auf einem Schiff seekrank werden kann“) wäre also eindeutig fremdinitiiert und würde nicht als Indikator für ein selbstständiges Erkennen und Umsetzen der Begründungspflicht auf Seiten des Kindes behandelt.

Mittel des Begründens: In diesem Analyseschritt wurden alle selbstinitiierten kindlichen Begründungen unter Aspekten der Vertextungskompetenz daraufhin betrachtet, wie komplex die argumentativen Verfahren jeweils sind (lokale Begründungen gegenüber komplexeren Einwänden, Abwägen mehrerer Begründungen etc., vgl. Quasthoff \& Domenech 2016).

Eine derart detaillierte Sequenzanalyse zur Platzierung und Durchführung argumentativer Verfahren legt auch offen, ob Elternteil (oder Kind) schon im Entscheidungsdiskurs (s. o. Abschnitt 3.2) dafür sorgt, dass argumentativ vorgegangen wird. Häufig werden z. B. Begründungen erst abschließend mit Bezug auf die Aufgabenstellung nachgeschoben, sodass sie im Rahmen des Aufgaben ${ }^{4}$ - und nicht des Entscheidungsdiskurses kontextualisiert sind.

4 Die Aufgabenstellung betonte die Gemeinsamkeit der Bearbeitung sowie eine anschließende Begründung. 
Zum Entscheidungsdiskurs (s. o. Abschnitt 3.2) zählen dabei in den Daten lokale Präferenzäußerungen („Ich würde drei ${ }^{5}$ nehmen“) bzw. entsprechende Aufforderungen, sich für eine oder mehrere der vorgegebenen vier Optionen zu entscheiden (,Was meinst du denn?“). Es geht also um Verfahren wie Benennung, Ausschluss und Festlegung von favorisierten Entscheidungsmöglichkeiten sowie Abwägungen zwischen mehreren Möglichkeiten zur abschließenden Lösung, aber auch um das Insistieren auf eine im Diskurs getroffene Entscheidung („Ich finde drei aber trotzdem besser“), jeweils ohne argumentative Stützung oder Ableitung.

Einer lokalen Entscheidungssequenz sollte sowohl kontextuell erwartungsgemäß als auch explizit durch die Aufgabenstellung der Studie gefordert eine Argumentationssequenz folgen, in der die eingenommene Position für den Interaktionspartner entsprechend erläutert bzw. begründet wird. Durch Verfahren wie z. B. begründetes Widerlegen (,Das ist aber AUCH nichts für die ganze Familie“), Einwenden (,Wenn aber jemand seekrank wird?“), aber auch Einlenken („Da hast du auch wieder Recht“) bzw. sich überzeugen lassen, gestalten die Interaktanten den Entscheidungsprozess argumentativ.

$\mathrm{Zu}$ den Besonderheiten der Daten gehören insgesamt die prinzipielle Vergleichbarkeit der Interaktionen sowohl über den Längsschnitt pro ElternKind-Dyade als auch zwischen verschiedenen Dyaden, aber gleichzeitig die kontextuell unterschiedlichen Bedingungen der jeweiligen Interaktionsmuster.

Das erste Ziel dieser Sequenzanalyse war pro EZP zunächst die Beantwortung der Frage, inwieweit Kinder selbstinitiierte Argumentationssequenzen produzieren. Im längsschnittlichen Vergleich wurde dann gefragt, ob die Kinder im Rahmen der Jobs höhere und komplexere Anteile übernehmen, um so einen Nachweis über die individuelle Entwicklung des Kindes im Hinblick auf seine Selbstständigkeit im Gespräch zu erlangen.

Da das Muster Fordern und Unterstützen Zugzwänge nur gemäß dem tatsächlichen Unterstützungsbedarf des Kindes einsetzt (Finetuning, s. o. Abschnitt 3.2), ist es gerade im Hinblick auf die kindliche Kompetenzentwicklung im Rahmen der Berücksichtigung des Kontextes notwendig herauszuarbeiten, inwiefern sich elterlich produzierte Zugzwänge über den Längsschnitt hinweg an die erhöhte Kompetenz des Kindes anpassen. Dabei ist auch zu berücksichtigen, wie nicht angepasste unterstützende Impulse im Dialog vom Kind angenommen werden.

5 Die Optionen waren auf dem Handout, das den Beteiligten vorlag, nummeriert. 


\section{Ergebnisse: Fordern und Unterstützen als Erwerbsressource}

Im Folgenden zeigen und diskutieren wir jeweils typische Verläufe der ElternKind-Interaktionen über die drei EZPe unter den Bedingungen der Muster Fordern und Unterstützen (5.1) und Raumlassen und Akzeptieren (5.2; s. auch Abschnitt 3.2), um die subtilen Unterschiede in der Mechanik gerade auch ähnlicher Muster offenzulegen. Die ausgewählten Fälle sind dabei für die längsschnittlichen Verläufe typisch, auch wenn die gezeigten Ausschnitte nicht immer prototypische Verfahren für das jeweilige Muster aufweisen. Im letzten Abschnitt 5.3 wenden wir uns den Daten in der Gesamtschau zu und berichten über die Verteilung beobachteter Veränderungsformen im Gesamtkorpus (vgl. auch Quasthoff et al. 2015; Heller \& Krah 2015).

\subsection{Ein typischer Verlauf im Rahmen von Fordern und Unterstützen}

Einleitend sei vorausgeschickt, dass die erschöpfende Auswertung des gesamten längsschnittlichen Korpus in FUnDuS die Stabilität der Interaktionsmuster als familiale Praktik bestätigte: Die drei familialen Interaktionsmuster Fordern und Unterstützen, Raumlassen und Akzeptieren und Übergehen und Selberlösen charakterisierten jeweils sowohl die mündlichen Entscheidungsals auch die Revisionsaufgabe (s. o. 3.1) und wurden in den Familien über den Längsschnitt im Wesentlichen beibehalten (vgl. Quasthoff et al. 2015). Die in FUnDuS rekonstruierten Interaktionsmuster scheinen also trotz des elizitierten Charakters der Daten (s. Quasthoff in diesem Band) sehr stabile familiale Praktiken abzubilden.

Wir zeigen zunächst den typischen längsschnittlichen Verlauf einer ElternKind-Dyade, die das Muster Fordern und Unterstützen praktiziert $(\mathrm{ET}=$ Mutter, $\mathrm{KI}=\mathrm{Sohn}$ ). Es handelt sich hier nach den gemessenen Kriterien der Stichprobenzusammensetzung um einen erwartungskonformen Fall mit hohem sozioökonomischen Status und hoher Argumentationskompetenz des Kindes.

Das folgende Beispiel (1.1) gibt einen Einblick in die kindliche Kompetenz zu Beginn des Längsschnitts. 
Beispiel 1.1: Mündliche Entscheidungsaufgabe ${ }^{6}$ Fall 1001: Fordern und Unterstützen_EZP 1

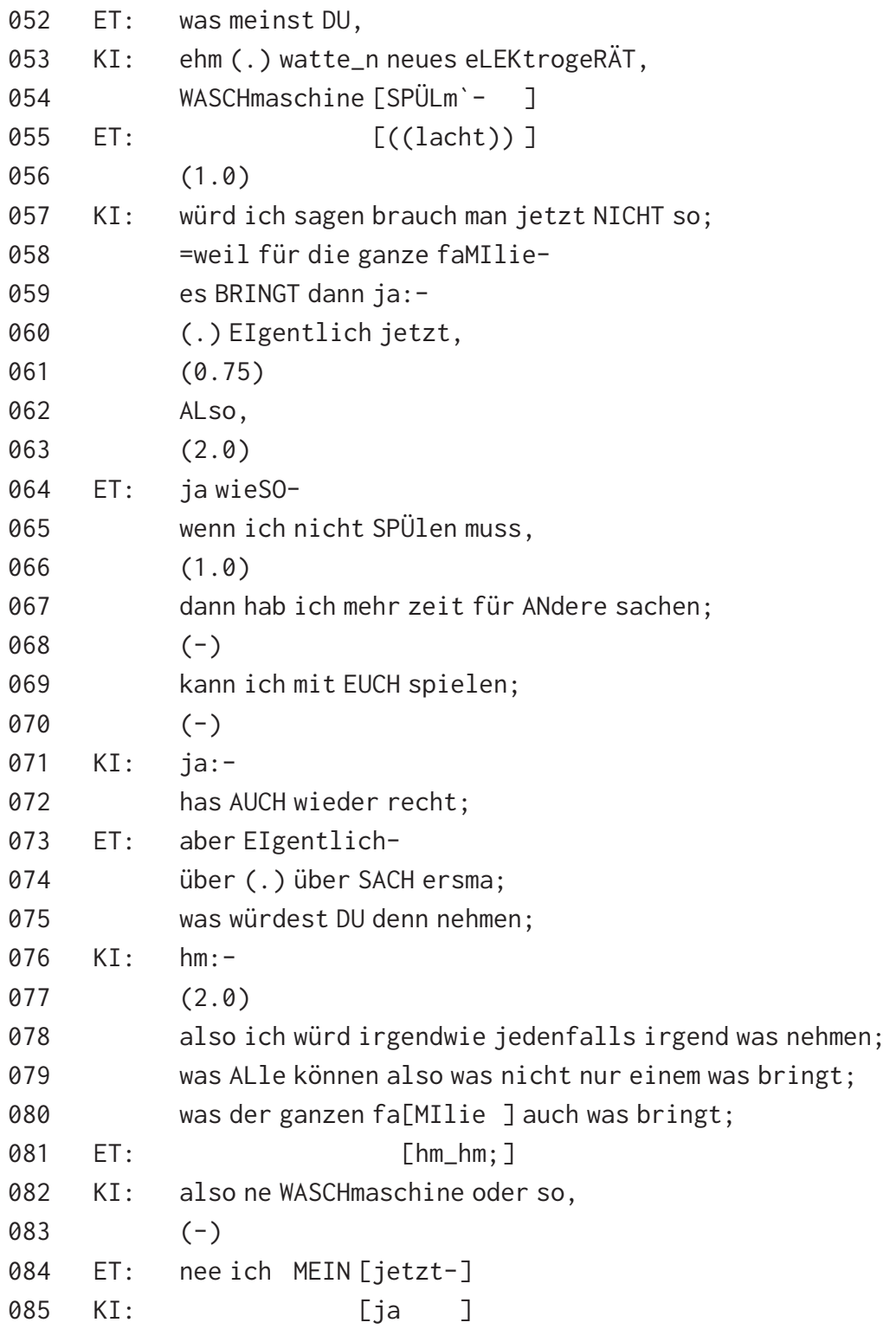

6 Entscheidung zwischen vier Gutscheinen. 
086

[ja ja KLAR; ]

087

088

089

090

091

092

093

094

095

096

097

098

099

100

101

102

103

104

105

106

107

108

109

110

111

112

113

114

115

116

117

118

119

120

121

122

123

ET: [von den vier] GUTscheinen; jetzt mal ganz spontan; was würdest du denn NEHmen;

$(-)$

$\mathrm{KI}: \quad \mathrm{hm}:-$

$(-)$

ET:

ich würd ne SPIElekon<<lachend $>$ sole $>$ [nehmen ];

KI: ja;

ET: nee;

ICH hätte jetzt toTAL;

(. ) auf das WOchenende getippt;

äh (. ) in einem FREIzei tpark;

KI: ja_a;

(2.0)

ET: ach ne SPIElekonsole;

MEINste,

KI : ja;

ET: wir HAM doch eine;

KI : ja;

(1.5)

has RECHT;

(1.5)

ähm

(2.0)

ET: und was is hier mit [dem BÜcher] dvd paket,

$\mathrm{KI}$ : $\quad$ [bücher ja ]

$\mathrm{hm}$ :

$(-)$

ET: ich sach ma für FÜNFhundert euro da kriste ja${ }^{\circ} \mathrm{hh}$

so: vIEle,

$(-)$

das SCHAFfst_e ja kaum;

(2.0)

$\mathrm{hm}$,

124

KI : joa;

125

das sind viel zu VIEle;

wenn schönes WETter is, 


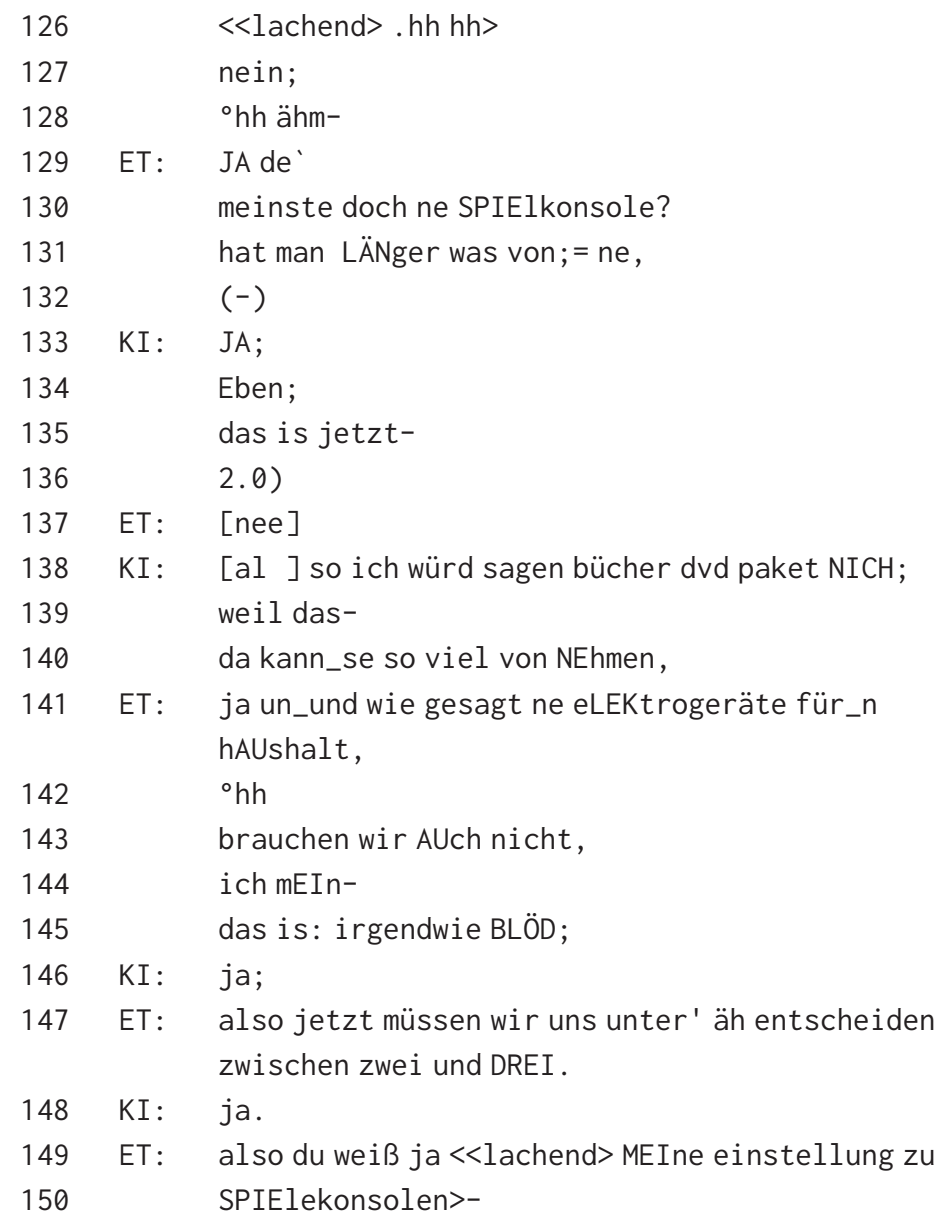

Charakteristisch für das Muster Fordern und Unterstützen ist bereits zu Beginn, dass das Elternteil das Kind gesprächsorganisatorisch als primären Aufgabenlöser etabliert, indem es das Kind gleich einleitend nach seinem Favoriten befragt (Z. 75; auch später in: Z. 87ff., 102, 130), bevor es selbst Präferenzen äußert. Kontrastiv sei auf einen Fall (726) von Übergehen und Selberlösen verwiesen, in dem der Dialog so beginnt:

Beispiel 2: Mündliche Entscheidungsaufgabe_Fall 726_Übergehen und Selberlösen

$001 \mathrm{KI}: \mathrm{hm}$;

002 ET: ich bin dafür zum beispiel, 
003 =ein wochenende für die ganze familie, weil dann können wir-

Das Kind im Beispiel (1) von Fordern und Unterstützen kommt diesem gesprächsorganisatorischen Zugzwang des Elternteils nach, gestaltet ihn aber anders aus, indem es nach dem Ausschlussverfahren vorgeht, statt seinen Favoriten zu nennen (Z. 53-59). Daraufhin bezweifelt das Elternteil die selbstinitiierte, jedoch nicht elaborierte Begründung des Kindes für den Ausschluss (Z. 58-60). Das Kind nutzt diese Anschlussmöglichkeit im Rahmen des Kernjobs Begründen im weiteren Verlauf nicht zur Elaboration seiner Begründung, sondern nimmt den Einwand vom Elternteil an (Z. 72).

Die anschließende Wiedereinsetzung des ursprünglichen gesprächsorganisierenden Zugzwanges des Elternteils (Z. 87-89), auf einer informelleren Ebene (jetzt mal ganz spontan) entspricht dem Discourse Acquisition Support System (s. o. Abschnitt 2; Hausendorf \& Quasthoff 1996) und verdeutlicht, dass das Kind aus der Sicht des Elternteils bislang nicht adäquat auf den globalen gesprächsorganisatorischen Zugzwang eingegangen ist. Dabei würdigt das Elternteil den gesprächsorganisatorischen Beitrag des Kindes (Nennung eines Hauptkriteriums: Z. 78-80) - nicht mustertypisch - nur minimal (Leßmann in diesem Band) und nutzt ihn auch nicht zur weiteren Organisation des Entscheidungsprozesses.

Angesichts der Tatsache, dass das Kind im Verlauf der Diskussion kaum selbstständig Begründungen für oder gegen eine Option vorbringt und meist auf einer lokalen Entscheidungsebene (Z. 82ff., 93, 104) verbleibt, steuert das Elternteil die Begründungen selbst bei (Z. 105, 120f., 131). Deutlich wird dies nach dem begründeten Einwand des Elternteils (Z. 105) gegen den vom Kind erwähnten Favoriten. Diesen entkräftet das Kind nicht, obschon dies erwartbar wäre. Das Kind verbleibt auf der Entscheidungsebene, indem es lediglich zustimmt (Z. 106-111). Argumente des Kindes sind zumeist Paraphrasen vorheriger elterlicher Äußerungen (Z. 116-118 vs. Z. 124f., Z. 138-140) und stellen damit eine vom Elternteil fremdinitiierte Begründung dar. Obwohl das Elternteil viel Raum für Begründungen lässt und das Kind als primären Aufgabenlöser etabliert, gelingt dem Kind kein selbstinitiierter Übergang in den Argumentationsdiskurs. Ferner ist zu sehen, dass das Elternteil maßgeblich das Gespräch formal organisiert, z. B. indem es bilanziert (Z. 147f.). Die minimale Beteiligung des Kindes am Argumentationsdiskurs korrespondiert damit, dass das Elternteil noch vergleichsweise wenig zum Nachliefern von Begründungen - etwa durch Warum-Fragen - anregt. In 
dieser Sequenz produziert das ET eher demonstrierend Argumente in Frageform (Z. 120, 131, 145). Durch dieses Verfahren bekommt das Kind die Möglichkeit, vorgebrachte Begründungen zurückzuweisen, zu ergänzen bzw. sie zu übernehmen. Das Kind tut in diesem Abschnitt Letzteres (Z. 123-126, 132ff.).

Entsprechend entsteht die thematisch und methodisch interessante Frage (s. o. Abschnitt 1), ob das Elternteil sich mit diesem Verfahren i. S. v. Fordern und Unterstützen angemessen auf das kindliche Kompetenzniveau einstellt, d. h. nur so viel konversationelle Mehrarbeit leistet, wie angesichts der (mangelnden) Beiträge des Kindes zur Aufrechterhaltung der (argumentativen) Interaktion notwendig ist (Finetuning), oder ob es mit dieser Praxis $\mathrm{zu}$ viel selbst übernimmt und das Kind damit dialogisch ,unterfordert‘ (Quasthoff 2011). Methodisch ist bei der Klärung dieser Frage auf der Grundlage der FUnDuS-Daten zu berücksichtigen, dass Eltern und Kind natürlich im Rahmen vorgängiger familialer Kommunikation Routinen entwickelt haben, in die die intuitiven Erfahrungen der Eltern zur kindlichen Partizipationsbereitschaft und -kompetenz eingegangen sein dürften. ${ }^{7}$ Um zu überprüfen, ob sich das elterliche Verhalten mit zunehmendem Alter des Kindes ändert sind wir hier also auf die uns vorliegenden weiteren zukünftigen Entwicklungen bis Klasse 9 angewiesen.

Allerdings finden wir im vorliegenden Beispiel (1) auch zum EZP 1 bereits einen Hinweis darauf, dass das Kind globalen Zugzwängen eher nicht nachkommt: In Z. 112 bietet das Elternteil durch eine Thematisierung einer Alternativoption (und was is hier mit [dem BÜcher] dvD paket) mit einem globalen Zugzwang die Anschlussmöglichkeit für eine Begründung, zumindest jedoch für eine weitere Entscheidungssequenz auf Seiten des Kindes. Das Kind gerät hierauf ins Stocken, weswegen das Elternteil mindestens an dieser Stelle durchaus entsprechend dem DASS erst nach Ausbleiben eines entsprechenden Zugs auf Kindseite selbst übernimmt (Z. 116-122).

Das Beispiel (1) steht also für die Fälle von Fordern und Unterstützen, in denen das Kind im EZP 1 zunächst nur eingeschränkt in der Lage zu sein scheint, ohne Unterstützung des Elternteils selbstständig insbes. Argumentationssequenzen zu produzieren, obwohl das Elternteil grundsätzlich die Möglichkeit für diese Aktivitäten bietet. Im weiteren Verlauf des Diskurses, der hier nicht wiedergegeben ist, verfährt das Elternteil wiederum prototypisch für das Interaktionsmuster Fordern und Unterstützen, indem es orientiert an den kindlichen Zügen lokalisierende Zugzwänge produziert. Entsprechend erhöhen sich

7 Dies unterscheidet diese Daten etwa von denen in Hausendorf und Quasthoff (1996), wo Kind und Erwachsene keine gemeinsame Interaktionsgeschichte hatten. 
zwar die Anteile des Kindes, besonders im Argumentationsdiskurs, aber diese sind aufgrund der Unterstützungsleistung fremdinitiiert, insofern sie eine Folge der elterlichen kontextuellen Hilfen darstellen. In der hier wiedergegebenen Sequenz lässt sich im Übrigen noch ein Unterschied zwischen Entscheidungsund Argumentationsdiskurs beobachten: Das Elternteil ratifiziert eher die vom Kind produzierten Entscheidungssequenzen, was sich auch auf die kindliche Partizipation am Entscheidungsdiskurs auswirkt, während es im Argumentationsdiskurs eher selbst begründet und einwendet, also übernimmt und damit Modelle bietet (Quasthoff 2011). Auf diese Weise wird dem Kind auch implizit eine eher niedrige Argumentationskompetenz zugeschrieben.

Beispiel 1.2: Mündliche Entscheidungsaufgabe ${ }^{8}$ _Fall 1001_Fordern und Unterstützen_EZP 2

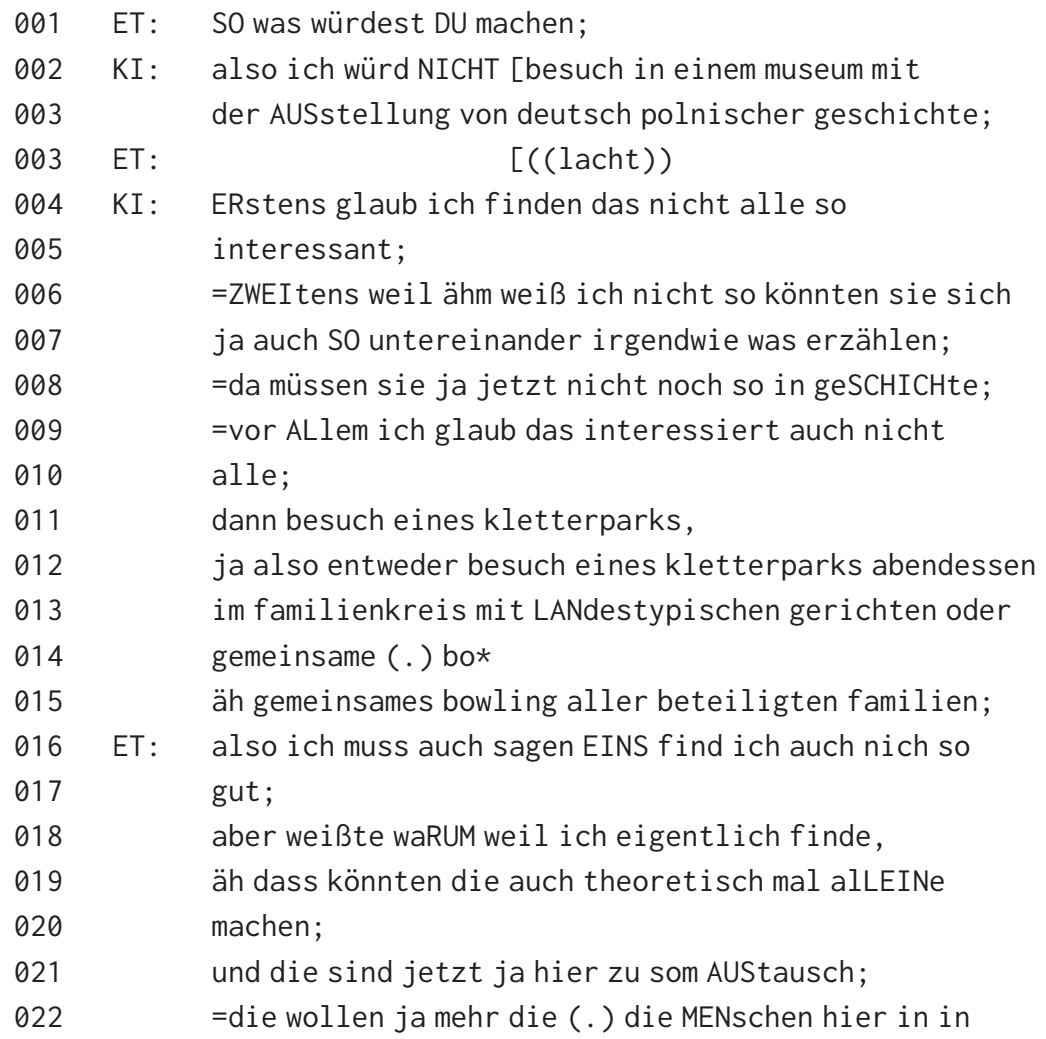

8 Entscheidung zwischen vier Freizeitaktivitäten mit Austauschschüler/innen. 
023

024

025

026

027

028

029

030

031

032

033

034

035

036

037

038

039

040

041

042

043

044

045

046

047

048

049

050

051

052

053

054

055

056

057 deutschland KENnen lernen;

$\mathrm{KI}: \quad \mathrm{ja}$;

ET: und son museum könnten sie ja $\mathrm{AUCH} \mathrm{h}^{\circ}$ weiß ich nicht mit ihren ELtern in polen oder alleine besuchen;

KI: ja gut wenns DAnach ging dann könnte man auch äh dann würde Abendessen im familienkreis mit landestypischen geRICHten; ich glaub nämlich nicht dass die dann irgendwie ähm

(. ) wie heißt sie BAYrisch creme oder so was dann in POLen essen;

ET: ja;

mit dem KLEtterpark,

KI : da würde man sich gegenseitig so KENnen lernen (. ) irgendwie ne,

ET: $\quad$ ja vor allem das GIBTS ja gar nicht überall ähm; könnt ich mir VORstellen dass denen ja wie gesagt dass die auch sagen (.) MENsch als wir damals in deutschland waren da ham wir son KLEterpark besichtigt; BOWlen tja weiß ich auch nicht; (1.1)

ET: also ich DENK [auch;

KI : $\quad$ [obwohl andererseits beim kletter PARK (-)

DA ist man ja immer so für sich alleine;

ET: $\mathrm{JA}$;

KI: das ist ja nicht so dass man jetzt so mitn ANdern; also beim BOwlen da wärs dann ja besser da könntest; du noch mi teinander äh über witzige geSCHICHTen reden (1.0) oder so;

ET : hm_hm; (1.2)

ET: $\quad$ [aber ;

KI : [also ich würd beSUCH eines kletterparks irgendwie auch WEG packen;

ET: stimmt [das macht man mehr EINzeln und [äh nicht 


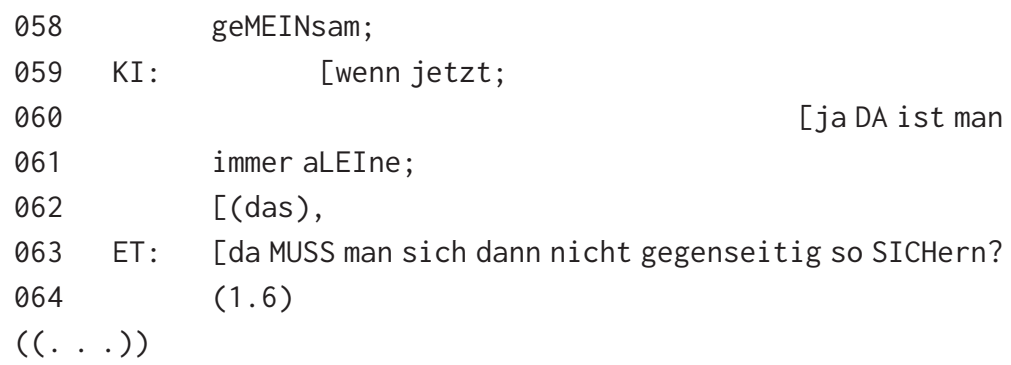

Die Aushandlung der 2. Erhebungswelle beginnt ebenfalls mit einem globalen gesprächsorganisatorischen Zugzwang des Elternteils (Z. 1). Das Kind kommt wie auch in EZP 1 - diesem Zugzwang nach, indem es jedoch zunächst Optionen ausschließt. Wiederum produziert das Kind jetzt unaufgefordert eine ausgebautere Argumentationssequenz, mit der es die Entscheidung gegen diese Option begründet (Z. 5-9). Unmittelbar anschließend etabliert das Kind diesen Ausschluss gesprächsorganisatorisch als Konsens, indem es nur noch die drei verbliebenen Optionen aufführt (Z. 11-15). Das Elternteil ratifiziert diesen Ausschluss, indem es eine weitere Begründung für den Ausschluss nachschiebt (Z. 16-23). Das Kind nimmt dann die Begründung der Mutter auf und erhebt sie zu einem Entscheidungskriterium, demgemäß es dann eine Präferenz äußert (Z. 27-32), die auf diese Weise elaboriert begründet ist. Die nächste Option wird von der Mutter genannt (Z. 34) und vom Kind seinerseits - anders als in einem vergleichbaren Kontext in Beispiel (1.1) - als passende Option initiativ begründet (Z. 35f.), worauf die Mutter eine weitere ausführlichere Pro-Begründung anschließt (Z. 30-34), gegen die aber das Kind einen Einwand erhebt (Z. 45f., 48-51), was schließlich zum konsensuellen Ausschluss dieser Option führt (Z. 55-64).

Es zeigt sich, dass der Siebtklässler in EZP 2 vermehrt selbstständig entscheidet und argumentiert, obschon die gesprächsorganisatorische Initiierung und Beendigung der Aushandlung noch mit EZP 1 vergleichbar ist. Besonders interessant ist die Initiierung eines Gegenarguments durch das Kind in Overlap (Z. 44f.), die zeigt, dass das Kind hier unabhängig von der elterlich produzierten thematischen Anschlussmöglichkeit in der Lage ist, ein Argument eigenständig zu vertexten und als nachgeschoben zu markieren. 
Beispiel 1.3: Mündliche Entscheidungsaufgabe ${ }^{9} \_$Fall 1001_Fordern und Unterstützen_EZP 3

\begin{tabular}{|c|c|c|}
\hline 038 & ET: & okay. \\
\hline 039 & & $(---)$ so. \\
\hline 40 & & was MEInst du? \\
\hline 41 & $\mathrm{KI}:$ & also auf jeden fall nicht das ERste und ZWEIte, \\
\hline 12 & ET: & $<<$ lachend $>$ das SEH ich auch so $>$. \\
\hline 13 & $\mathrm{KI}:$ & weil (-) ERstens ist es vielleicht nicht so ganz \\
\hline 14 & & interesSANT für die ganze klasse, \\
\hline 45 & & und vor allem find ich bei ner abschlussfahrt sollte \\
\hline 46 & & man vielleicht noch mal SPAß haben; \\
\hline 47 & & das HEIßt (-) irgendwie kulTURprogramm oder musical \\
\hline 48 & & ist jetzt nicht so das WAHre; \\
\hline 49 & & ich würd sagen das VIERte. \\
\hline 50 & ET: & also ich denk auch das erste und ZWEIte, \\
\hline 51 & & (.) das ist mehr was für erWACHSene. \\
\hline 52 & & also ich könnt mir nicht vorstellen, \\
\hline 53 & & ihr SEID (.) vierzehn fünfzehn. \\
\hline 54 & & dass MUSICAL sowas für EUch ist. \\
\hline 5 & & und ROM kulTURprogramm (-) denk ich ist auch eher was \\
\hline 56 & & für erWACHSene. \\
\hline & & jetzt [ist \\
\hline 58 & $\mathrm{KI}:$ & [was ist denn hier noch mit SEgeltörn gemeint? \\
\hline & ET: & ja auf dem IJSselmeer- \\
\hline & & mietest du dir nen schiff wird ein SCHIFF gemietet; \\
\hline & & wahrscheinlich ist der kapiTÄN oder paar besatzungs \\
\hline 52 & & mitglieder sind daBEI, \\
\hline 63 & & und man selber muss ein bisschen MIThelfen aber man \\
\hline 54 & & man ist ja halt aufm SCHIFF. \\
\hline 65 & $\mathrm{KI}:$ & JA: ANdererseits wenns da ein paar gibt die irgendWIE \\
\hline 66 & & SEEkrank oder so werden, \\
\hline & & find ich das VIERte vielleicht doch noch besser; \\
\hline & & weil du da auch mehr (. ) MÖGlichkeiten hast. \\
\hline & & oder nicht? \\
\hline & & ich meine wenn du da fünf TAge bist, \\
\hline & ET: & ja vor allem man ist FÜNF TAge auf dem Schiff. \\
\hline
\end{tabular}

9 Entscheidung zwischen vier Zielen für eine Klassenfahrt. 
$071 \mathrm{KI}:$ ja ja,

072 ET: man weiß auch NICHT ich meine ihr als klasse ihr

073 kennt EUCH,

074 (. ) und ihr seid ja schon weiß ich nicht jetzt fünf

075

076

077

078

079

080 jahre oder so zuSAMmen, aber die ELtern die kennen sich ja nicht so gut.

$\mathrm{KI}$ : und wenn jetzt die ELtern [ha'

[fahren die eltern

087

088

089

090

091

092

093

094

095

096

097

098

099

$\mathrm{KI}$ : (warte) überhaupt MIT?

(1.0)

ET: ach SO,

WARte mal eben.

$(--)$

KI: naja ist ja eGAL aber trotzdem=

ET: =NEE die eltern FAHren gar nicht mit.

$(($ lacht $))$

KI: $\quad<<$ grinsend $>$ ja dann ist ja eGAL $>$.

ET: ((lacht))

ich hatte gedacht die ELtern fahren mit.

[ne hast REcht

KI : [ja aber TROTZdem wenns da son paar gibt so fünf leute oder so die SEEkrank sind;

ist das jetzt für die vielleicht nicht so optiMAL,

ET: ja und man HÄNGT natürlich auch die fünf tage mehr oder weniger zuSAMmen.

ne?

=man kann NICHT mal sich son BISSchen (.) so groß sind die SCHIFFe ja nicht[und äh

KI: [ja gut aufm (.) bei ner ABschlussfahrt möchtest du jetzt ja auch nicht so viel alleine machen aber-

$108 \mathrm{KI}: \mathrm{ja}$,

109 ET: also [ich würd

$110 \mathrm{KI}$ : [ich find das VIERte auch am besten, 
111

115

116

117

118

119

120

122

123

124

125

126

127

128

129

130

131

132

133

134

135

136

137

138

139

140

141

142

weil dann (. ) sich son paar keine ahnung da kann man

auch verSCHIEdenes machen, weil wenn man jetzt sich so zum spiel nur so

kulTURprogramm einigt, und son paar einfach überhaupt kein BOCK zu haben;

da kannse bei äh keine ahnung CENterrparcs oder wo man da hingeht vielleicht noch sowas verSCHIEdenes machen.

das zum beispiel ZEHN gehen schwimmen;

ZEHN gehen dann shoppen oder sowas.

dann machse ja TROTZdem noch was zusammen.

ET: also ich würd auch sagen VIER allein schon ich sach mal die MÄDchen vielleicht das die eher sagen shoppen, die JUNGS sagen wir gehen schwimmen; und man kann (. )TROTZdem ich denk mal abends da wirds vielleicht irgendne BOWlingbahn oder irgendwie sowas GEben .

dass man DANN eben sagt vormittags (. ) können immer vier oder fünf in grüppchen was EINzelnes machen, und irgendwie NACHmittags oder abends wir machen alle $(-)$ [zwanzig oder so was ne?

KI : $\quad$ [ja wie gesagt. und ich find vor allem (eine) ABschlussfahrt sollte nochmal SPAß machen, und so zeit wo wa was zuSAMmen machen; und wenn du in so nem MUsicalbesuch bist oder sowas wo du einfach nur zwei (. ) bis zweieinhalb STUNden da SITZT, und einfach nach VoRne guckst; ist jetzt vielleicht nicht so toll. würd ICH das vierte (nehmen)

Die Aushandlung vom EZP 3 beginnt wiederum mit derselben Grobstruktur wie bei EZP 1 und 2: Das Elternteil fordert das Kind auf, Präferenzen zu äußern, das 
Kind schließt stattdessen Optionen begründet aus, das Elternteil ratifiziert diesen Ausschluss durch eine eigene Begründung (bis Z. 56). Allerdings beendet das Kind das Ausschlussverfahren diesmal gleich mit einer Favoritenwahl (Z. 49). Nach einer kindinitiierten Nebensequenz (Z. 58-64), in der es Informationen erfragt, schränkt es selbst die von ihm benannte Option (Segeltörn) als mögliche erste Wahl begründet ein (Z. 65-69). Unterstützende Argumente des Elternteils (Z. 72-77) führen zu einer Unklarheit mit Bezug auf die Vorgaben der Aufgabe und eine weitere vom Kind initiierte Nebensequenz (Z. 78f.).

Im weiteren Verlauf der Interaktion wird nun deutlich, dass nicht mehr das Elternteil das Kind gesprächsorganisatorisch durch den Entscheidungs- und Argumentationsdiskurs führt. Nach der Eröffnung des Elternteils übernimmt das Kind selbstständig und initiativ die Aufgabenlösung, indem es gesprächsorganisatorisch eine aktive Rolle einnimmt (Z. 86, 89, 110), z. B. durch die Initiierung von zu klärenden Informationen in Form von Nebensequenzen (Z. 58, 78f.). In Z. 109 deutet bspw. zunächst das Elternteil die Übernahme der Gesprächsorganisation an, indem es ein Zwischenfazit bzw. eine finale Entscheidung ankündigt (Z. 109: also ich würde?). Dies wird jedoch vom Kind (Z. 110) unterbrochen, das seinerseits seinen endgültigen Lösungsvorschlag festlegt und begründet (Z. 110-121). Damit reklamiert es die Rolle des Aufgabenlösers allein. Den konversationellen Job Abschließen übernimmt dann schlussendlich wieder das Elternteil (Z. $127 \mathrm{ff}$.).

Das Kind etabliert jetzt durchgängig selbstständig Argumentationssequenzen (Z. 111-114), konkretisiert und wendet begründet ein (Z. 93-95). Diese größeren argumentativen Anteile akzeptiert das Elternteil und schließt mit weiteren Begründungen an, die für die vom Kind vorgeschlagene Entscheidung sprechen (Z. 120, 123). So sind die Aushandlungen in dieser Dyade eher von Konsens und gegenseitiger Ergänzung zwischen Elternteil und Kind, weniger von Dissens geprägt. Die gemeinsame und äußerst kooperativ wirkende Exploration (Ehlich 2012) wird besonders im EZP 3 deutlich.

Die Analysen der drei Transkripte machten deutlich, dass auf elterlicher Seite durchgängig das Interaktionsmuster Fordern und Unterstützen mit seinen erwerbssupportiven Verfahren (s. o. Abschnitt 3.2) praktiziert wurde: Das Elternteil

- lässt Gesprächsraum für kindliche Gesprächsbeiträge;

- akzeptiert eine abweichende Position des Kindes (z. B. Z. 37, 44, 49 in EZP 2; Z. 92 in EZP 3);

- fordert das Kind im Falle noch unklarer inhaltlicher Aussagen zu weiteren Elaborierungen / Begründungen auf (z. B. Z. 105, 130 in EZP 1; Z. 25f. in EZP 2; Z. 96-101 in EZP 3);

- begründet durchgängig auch die eigene Position. 
Zur Frage des elterlichen Finetuning ist nun festzustellen: Während zu erkennen ist, dass das Kind in EZP 1 bei der Partizipation im Argumentationsdiskurs unmittelbaren Unterstützungsbedarf hat, welchen das Elternteil erst später im Verlauf des Gespräches bietet, zeigen die Analysen der elterlichen Gesprächssteuerung in der 2. und besonders der 3. Erhebung nun deutlich, dass sich das Elternteil tatsächlich entsprechend der zunehmend aktiveren Rolle des Kindes zurücknimmt. Dies geht in EZP 3 so weit, dass die gesprächssteuernde Rolle des Elternteils, an der sich das Kind noch in EZP 1 weitgehend und in EZP 2 bereits in geringerem Ausmaß orientiert, praktisch auf das Kind übergeht. Während das Elternteil noch im EZP 1 durch die Produktion von Zugzwängen den Verlauf des Gespräches und der Argumentation maßgeblich vorgibt und das Kind entsprechend fremdinitiierte Entscheidungen und Begründungen formuliert, besteht im Verlauf von EZP 3 die Notwendigkeit einer solchen Unterstützungsleistung nicht mehr und sie wird auch nicht mehr angeboten. Da das Kind nunmehr in der Lage ist, selbstständig Entscheidungen sowie Argumentationen kontextuell sinnvoll zu entfalten, fährt das Elternteil seine eigene ,Mehrarbeit‘ (Quasthoff 2011) entsprechend zurück, das Finetuning funktioniert also.

Die deutlich erhöhten Anteile des Kindes am Aufgaben-, Entscheidungs- und Argumentationsdiskurs sowie die ausgebauteren argumentativen Sequenzen lassen auf einen Kompetenzzuwachs des Kindes im Bereich der Kontextualisierungskompetenz und Vertextungskompetenz schließen. Die zunehmende Selbstständigkeit wird deutlich an der Erhöhung der kindseitig selbstständig initiierten Aktivitäten, z. B. fordert das Kind in EZP 3 Unterstützungsleistungen vom Elternteil explizit ein (z. B. Erkundigungen über den Aufgabenkontext sowie Erläuterungen über Begrifflichkeiten). An anderen Stellen lehnt es elterliche Unterstützung ab bzw. übergeht sie (Z. 107 in EZP 3). Es ist nun das Kind, das seinen Unterstützungsbedarf reguliert.

Auch die benutzten Verfahren sind - besonders im Entscheidungsdiskurs im EZP 3 - deutlich elaborierter als in EZP 1 und 2. Sie richten sich häufiger gegen erste Vermutungen des Elternteils, während kindliche Entscheidungsäußerungen in EZP 1 und 2 elterliche Äußerungen eher bestätigen. Auch agiert das Kind in EZP 3 deutlich globaler, indem es mehr Optionen in einer Äußerung gegeneinander abwägt, Schlussfolgerungen formuliert und Kriterien definiert, die eine Option erfüllen sollte, um als präferiert ausgewählt zu werden.

Dabei zeigt die kontextuell vergleichende Rekonstruktion der drei strukturell ähnlichen Interaktionen mit demselben Kind auf unterschiedlichem Altersniveaus, in welcher Weise sich die erhöhte Kompetenz des Kindes äußert und von der Mutter interaktiv akzeptiert wird. Dieser Fall von Fordern und Unterstützen demonstriert aber - im Unterschied zu dem folgenden zweiten - auch, in welcher Weise sich kompetenteres Verhalten des älteren Kindes mit interaktiven Erfahrungen des jüngeren in Verbindung bringen lässt: Es wird deutlich, inwieweit 
das Elternteil Erwerbsressourcen anbietet, zunächst durch interaktives ,Demonstrieren' (Hausendorf \& Quasthoff 1996) und später durch feinabgestimmte Unterstützung, was beides eine effektive Erwerbsressource darzustellen scheint.

\subsection{Ein typischer Verlauf im Rahmen des Musters Raumlassen und Akzeptieren}

Das Muster Raumlassen und Akzeptieren lässt dem Kind zwar Gesprächsraum, fordert es aber eher nicht dialogisch heraus und unterstützt es nicht, sondern akzeptiert eher und lässt es allein (s. o. Abschnitt 3.2; Heller \& Krah 2015). Unter dem Gesichtspunkt, dass die Interaktion in Beispiel (1) ebenfalls eher von wechselseitigem Akzeptieren als von herausforderndem Dissens geprägt ist, erwarten wir hier im Vergleich zu fordernden und unterstützenden Praktiken eher subtile - aber gerade deshalb besonders interessante - Unterschiede. Weil beide Muster - im Unterschied zu Übergehen und Selberlösen - dem Kind eine aktive Rolle zugestehen, dürfte die Ausgestaltung der Interaktion gemäß der Logik des Musters Raumlassen und Akzeptieren stark davon abhängen, wie das Kind seinen Gesprächsraum nutzt, d. h. wie kompetent und partizipativ es auch ohne anspruchsvolle Steuerung im Gespräch agiert. Der Fall 144 repräsentiert einen prototypischen Fall für Raumlassen und Akzeptieren. Auch diese Familie hat einen hohen sozioökonomischen Status.

Beispiel 3.1_Mündliche Entscheidungsaufgabe_Fall 144_Raumlassen und Akzeptieren_EZP 1

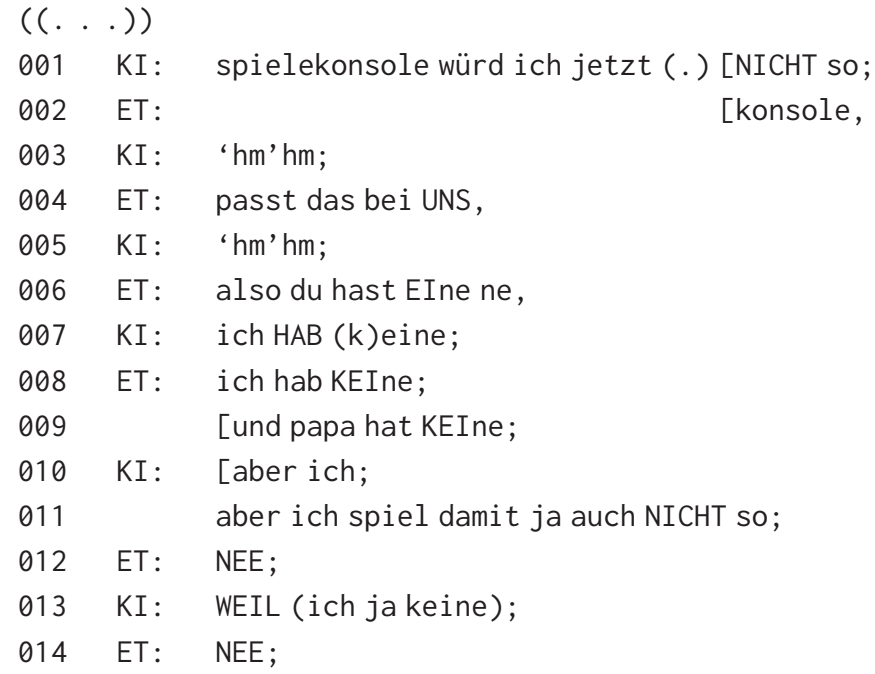




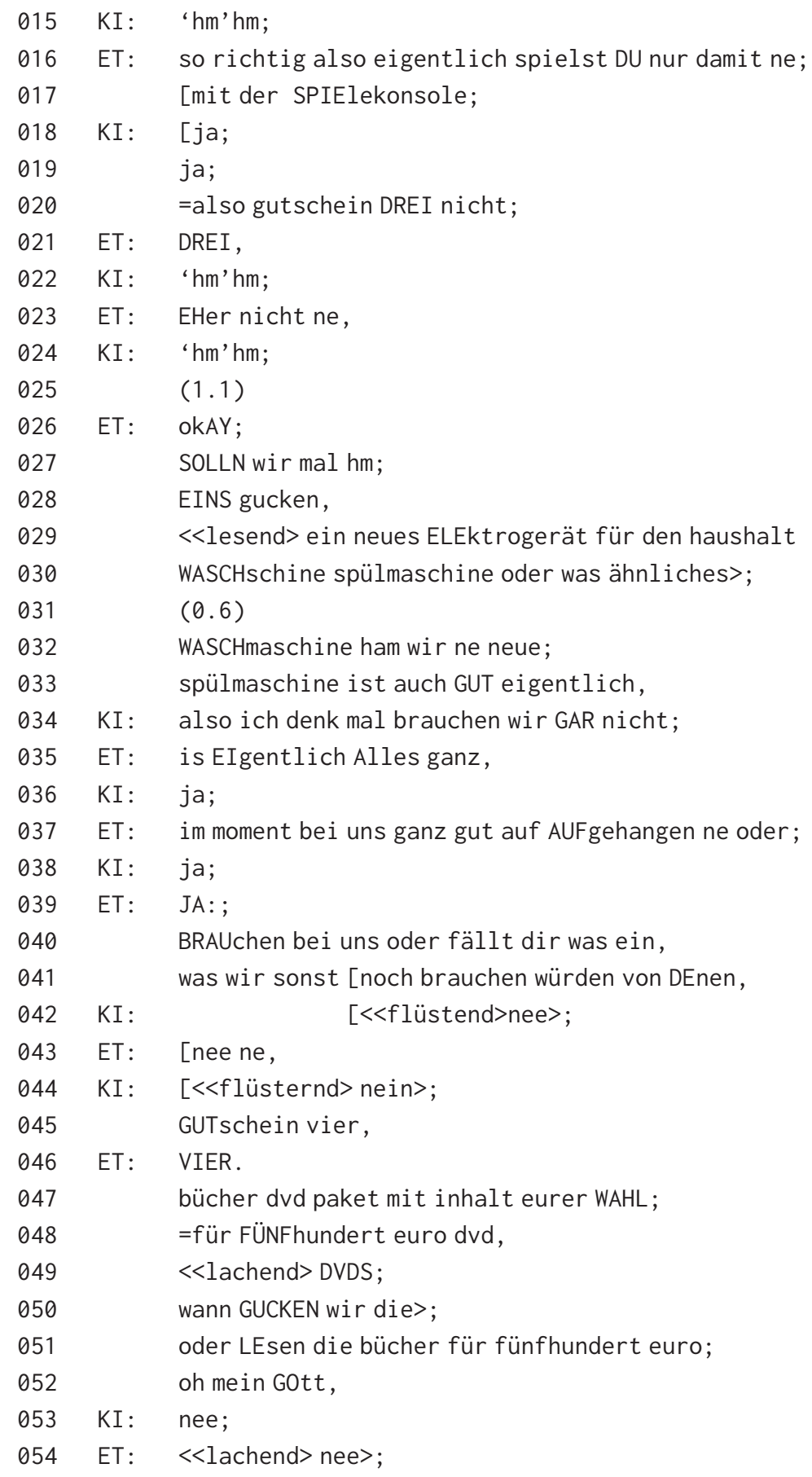




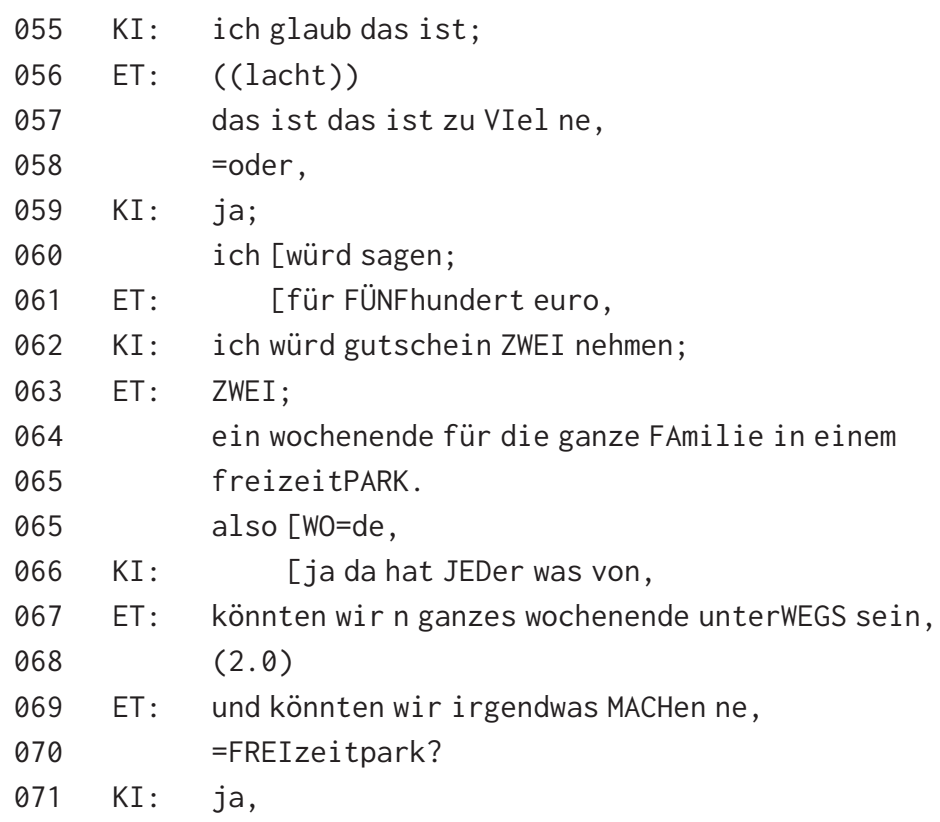

Die Aufnahme beginnt hier (nach einer Aufzählung der Optionen durch die Mutter) nicht - wie im Beispiel (1) - mit einer gesprächssteuernden Aufforderung an das Kind, sondern das Kind tritt seinerseits - wiederum unter Nutzung des Ausschlussverfahrens - in den Entscheidungsdiskurs ein, allerdings ohne Begründung. Das Elternteil liefert in Form von Entscheidungsfragen zwar Vorlagen für mögliche Begründungen (Z. 4, 6), die aber jeweils vom Kind nur minimale reaktive Züge (Z. 5, 7) verlangen. Das Kind steuert seinerseits eine neue Begründung für den Ausschluss einer Option bei (Z. 11: aber ich spiel damit ja auch nicht), die jedoch nicht zu Ende geführt wird (Z. 13), ohne dass dies zu entsprechenden Nachfragen des Elternteils führen würde.

Entsprechend der Charakteristik des Interaktionsmusters Raumlassen und Akzeptieren fordert das Elternteil das Kind durchgängig nicht, sondern schließt sich eher durch minimale eigene Begründungssequenzen (Z. 34: brauchen wir gar nicht; Z. 37, 50 f., 57) an die vom Kind genannten Optionen und Begründungen an. Die Interaktion wirkt dabei durchaus kooperativ, z. B. vervollständigt die Mutter eine vom Kind abgebrochene Äußerung (Z. 55) stellvertretend und überlässt dem Kind dabei durch die tag question (oder?) die Autorschaft (Z. 57: das ist das ist zu viel ne,=oder). Ein vergleichbarer Fall im Rahmen des Musters Übergehen und Selberlösen zeigt hier wiederum die Unterschiede zwischen den Mustern: Das Elternteil, das Übergehen und Selberlösen praktiziert, übernimmt im Unterschied zu Beispiel (3.1) die nicht 
produzierte Begründung des Kindes, ohne sie dem Kind zuzuschreiben (Z. 15: also ich würde schon sagen)

Beispiel 4 Mündliche Entscheidungsaufgabe_Fall 833_Übergehen und Selberlösen_EZP 1

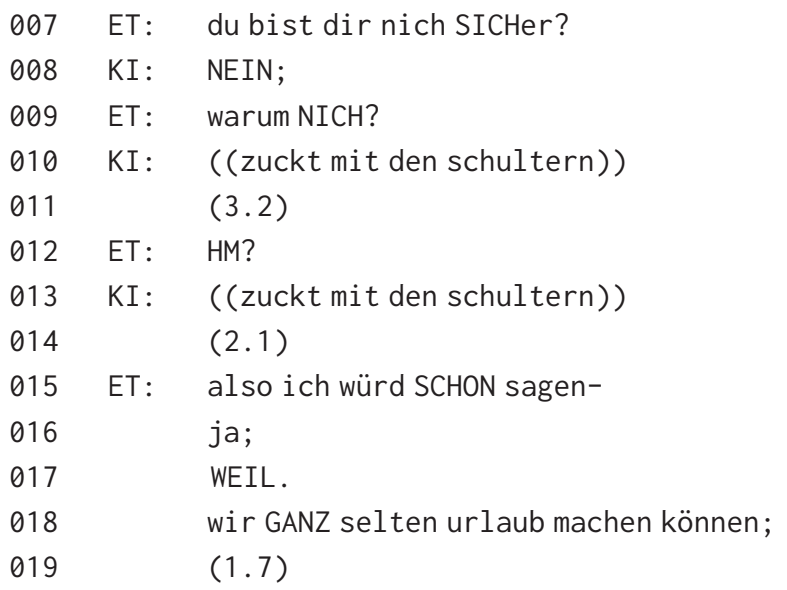

Die vervollständigende Elaboration von kindlichen Äußerungen kennen wir aus der frühen Forschung zu Motherese (Garnica 1977) bzw. child directed speech (Snow 1977) gegenüber sehr jungen Kindern, die noch im Prozess des Grammatikerwerbs sind, wodurch das Verfahren dort entsprechend erwerbsfunktional ist. Bei einer Sechstklässlerin, die ihre syntaktisch unvollständige Äußerung abbricht, wären als Erwerbsressource eher Zugzwänge zur größeren Explizitheit funktional.

Auch im Bereich der Gesprächsorganisation steuert das Elternteil zwar selbst (Z. 27 f., 40 f.), lässt dem Kind aber v. a. auch Raum für eigene Aktivitäten, den dieses auch nutzt (Z. 20, 45, 62).

Zusammenfassend finden sich im elterlichen Verhalten die Merkmale von Raumlassen und Akzeptieren wieder (s. o. Abschnitt 3.2): Das Elternteil

- bietet Raum für kindliche Äußerungen (z. B. Z. 37, 40 in EZP 1; Z. 60, 76 in EZP 2; Z. 13, 68-71 in EZP 3);

- die Ratifizierungen der Äußerungen des Kindes erfolgen eher beiläufig (z. B. Z. 23 in EZP 1; Z. 19 f., 23f., 35, 63f. in EZP 2; Z. 15, 19, 22f. in EZP 3);

- unterstützende Nachfragen oder Einwände werden allenfalls global formuliert und oft ganz unterlassen

(z. B. Z. 6, 32, 57 in EZP 1; 11, 51-54 in EZP 2; Z. 40, 65 in EZP 3);

- es findet entsprechend kein Finetuning statt (z. B. Z. 15, 63f. in EZP 1; Z. 31-33, 74f., 88 in EZP 2; Z. 26-28, 79-82 in EZP 3). 
Das Kind der 6. Klasse verbleibt i. Allg. auf einer lokalen Entscheidungsebene und begründet nur in Ausnahmefällen selbstinitiiert (Z. 11, 34, 66) mit jeweils wenig ausgebauten Argumenten. Es neigt dazu, begründende Äußerungen abzubrechen $(Z .13,55)$ und hat mit diesem impliziten Stil insofern Erfolg, als die Mutter die Andeutungen entweder in dieser Form ratifiziert (Z. 14) oder ihrerseits vervollständigt (Z. 56), aber jedenfalls nicht wegen Verständigungsproblemen eine Elaboration auslöst. Unter Erwerbsgesichtspunkten hätte das Kind also i.S. einer auszubauenden Argumentationskompetenz durchaus externe Ressourcen nötig, die ihm aber im Rahmen der elterlichen Interaktionspraktik im dokumentierten Gespräch kaum angeboten werden.

Beispiel 3.2_Mündlich e Entscheidungsaufgabe_Fall 144_Raumlassen und Akzeptieren_EZP 2

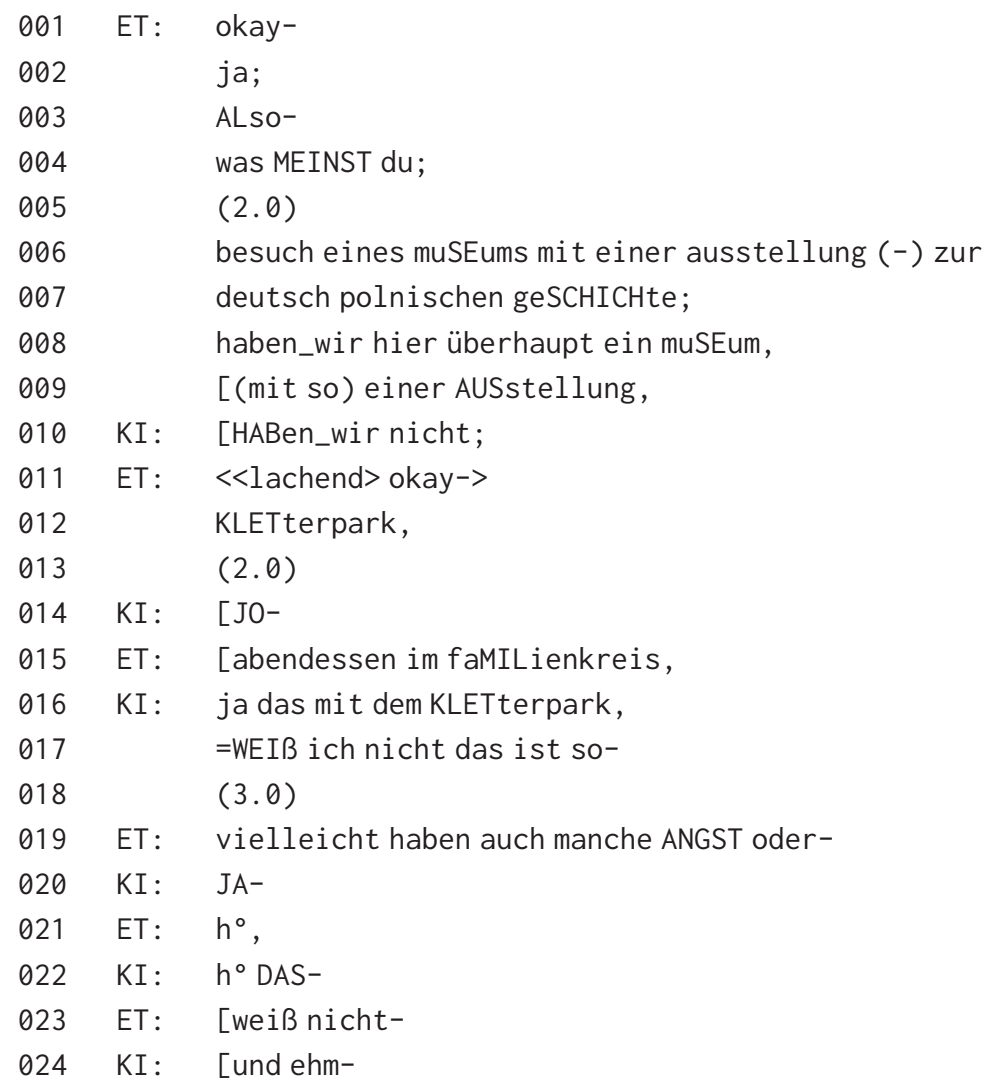




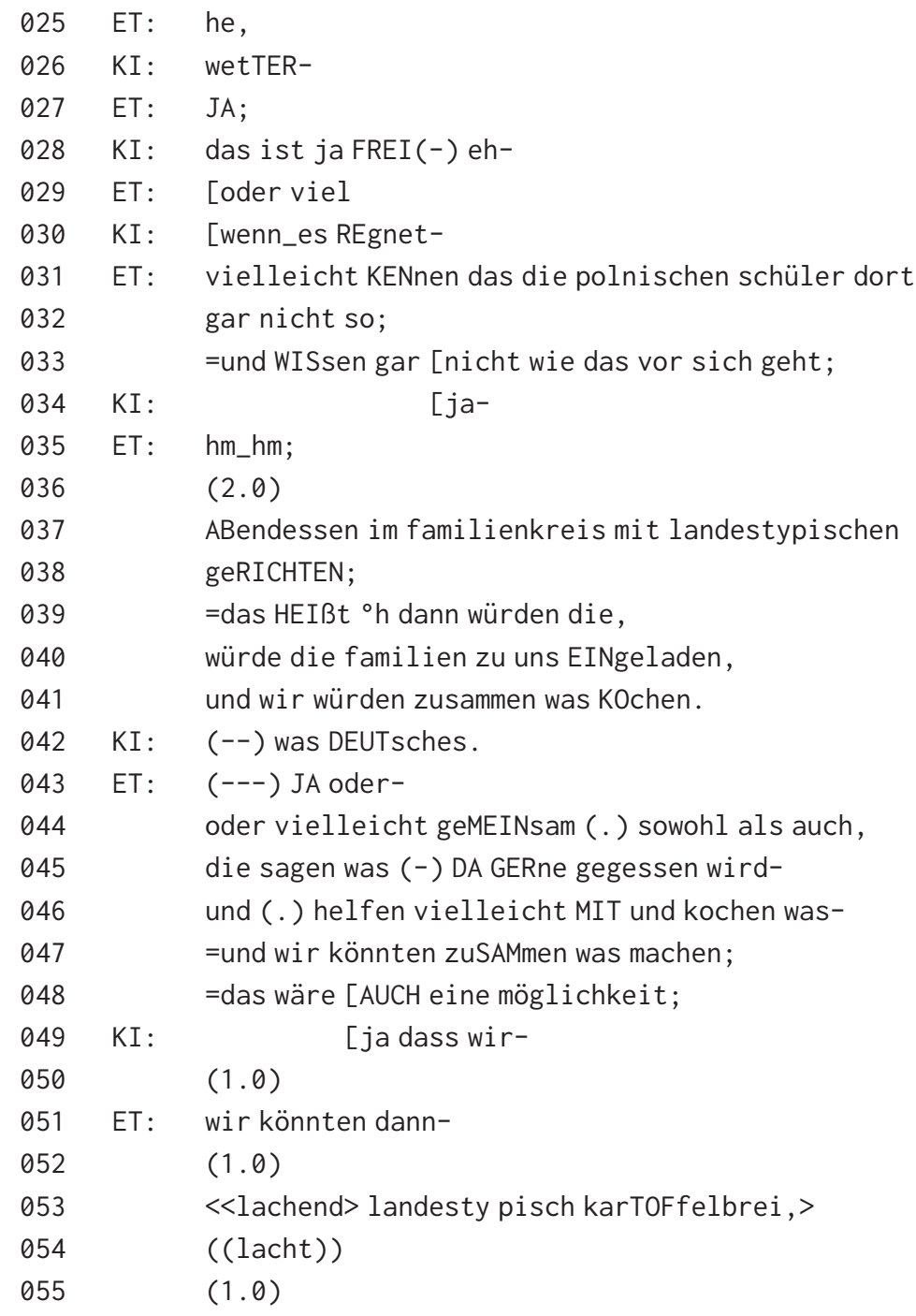
oder gemeinsames bowling ALler beteiligten familien; =das heißt mit ALlen die kommen, allen familienzum BOWlingcenter fahren.

KI : das find ich GUT,

063 ET: hm_hm- 


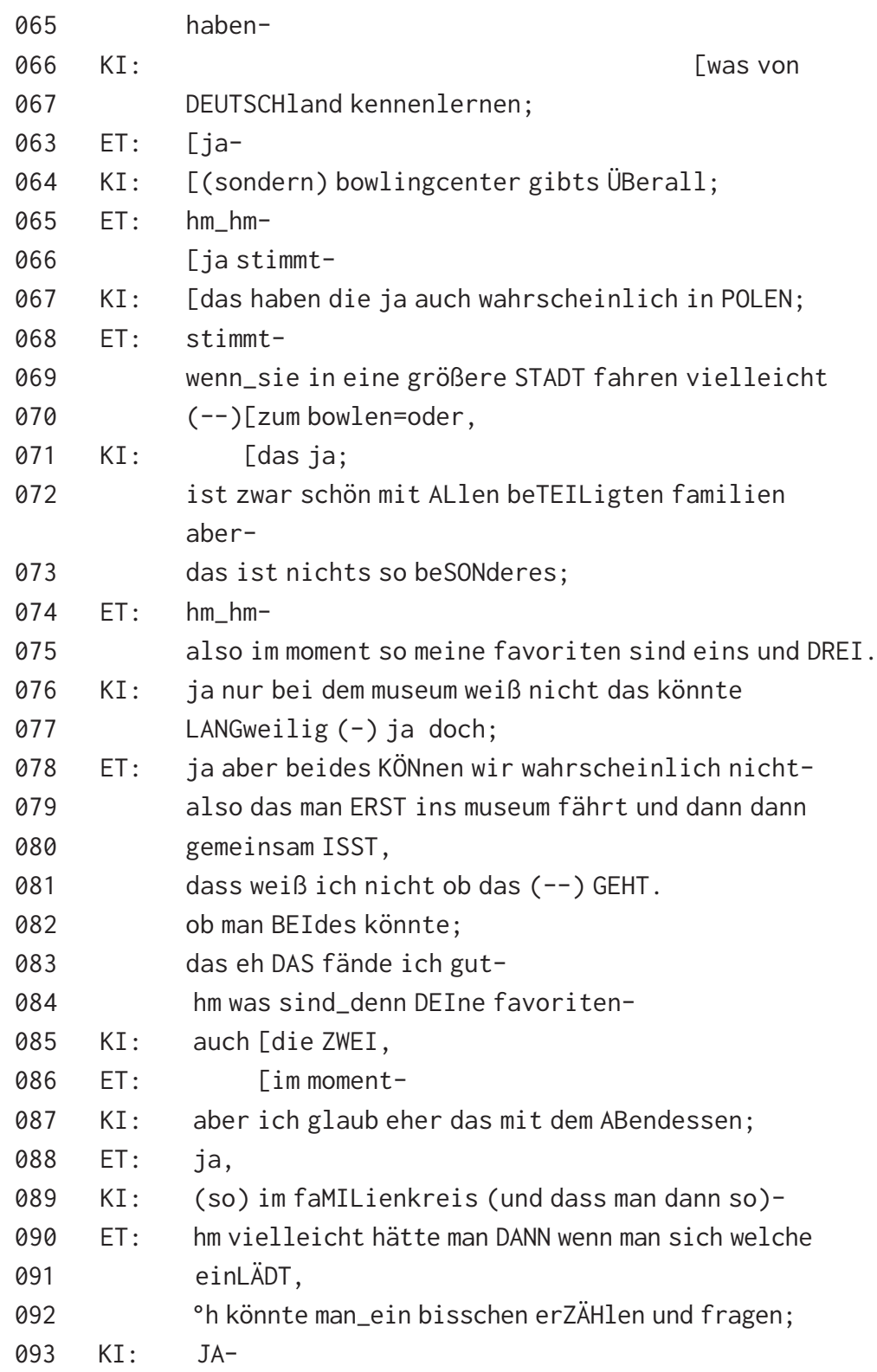

Das Elternteil beginnt diese Aushandlung (wie in Beispiel (1)) mit dem Zugzwang an das Kind, eine Präferenz zu äußern (Z. 4), was das Kind jedoch verweigert: (Z. 6). Die Mutter übernimmt dann, indem sie scheinbar willkürlich eine Option thematisiert (Z. 6f.) und anschließend eine Nebensequenz mit 
einer Frage über die konkrete Verfügbarkeit bzw. die generelle Realisierbarkeit der Option vor Ort initiiert (Z. $7 \mathrm{f}$.).

Diesmal thematisiert das Elternteil durchgängig gesprächssteuernd die weiteren Optionen (Z. 12, 15, 37f., 56). Auch im argumentativen Diskurs beteiligt sich das Kind nicht merkbar aktiver als zum EZP 1: Es gibt nur wenige selbstinitiierte Begründungen von KI (Z. 61f., 76f.), sie sind oft abgebrochen bzw. semantisch und syntaktisch nicht ausgebaut (Z. 17, 26, 28, 30), werden aber vom Elternteil nur z. T. expliziert (Z. 18f.). Eine Äußerung des Kindes (Z. 60: das finde ich gut) ist in ihrem anaphorischen Bezug uneindeutig, was aber ebenfalls nicht aufgeklärt wird. Allerdings arbeiten beide danach - ausnahmsweise elternseitig eingeleitet mit also du meinst (Z. 64) - länger und aufeinander bezogen am Ausbau eines Arguments des Kindes (ab Z. 61f.: und ich finde das hat nicht so viel so mit ( - ) DEUSCH(ländern) oder so zu tun (genuschel)-).

Dieses Gespräch zeigt, dass das Kind auch in der 7. Klasse Begründungen noch allenfalls lokal und oft nur angedeutet produziert. Die akzeptierende Haltung des Elternteils auch gegenüber impliziten oder in mehreren Anläufen vorgebrachten Argumenten (Z. 24-30), die oft einfach fallengelassen werden (Z. 31), erschwert einen Ausbau des Argumentationsdiskurses. Auch im Gesprächsmanagement nutzt das Kind den Raum, der ihm gelassen wird, eher noch weniger als in der ersten Erhebung. Ein Kompetenzzuwachs wird von EZP 1 zu EZP 2 nicht erkennbar.

Im Unterschied zu Fall 1001 wird das Kind hier also in seinem Gesprächsraum weitgehend allein gelassen. Es vergrößert diesen Raum nicht, wenn es älter wird, geschweige denn, dass es ihn mit ,neuen Möbelstücken‘, also neuen argumentativen Verfahren, anders einrichtet.

Beispiel 3.3_Mündliche Entscheidungsaufgabe_Fall 144_Raumlassen und Akzeptieren_EZP 3

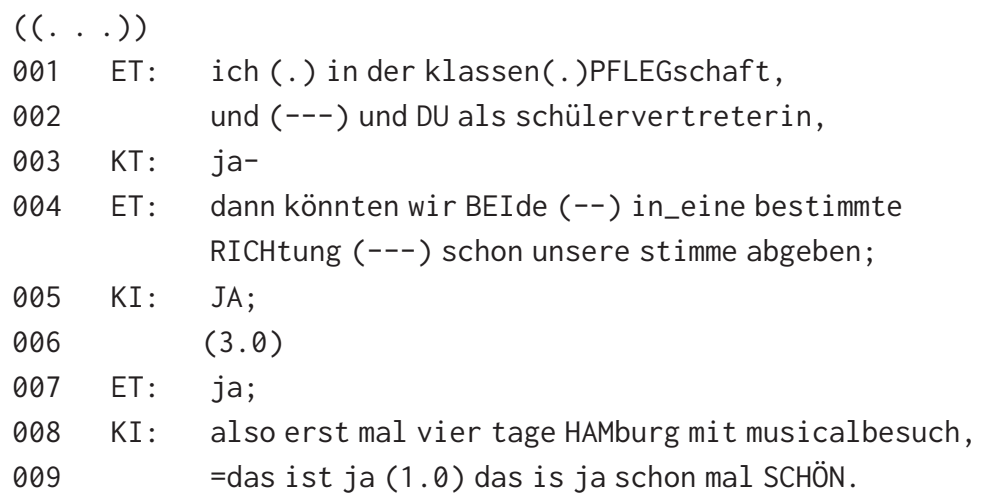


010 ET: jahört sich GUT an ne,

011 KI: drei tage ROM mit [kulturprogramm,

012 ET: [kulturprogramm,

$013 \mathrm{KI}$ : ichmein-

014 klar rom ist AUSland,

015 ET: hm_hm,

$016 \mathrm{KI}$ : das ist natürlich SCHÖN aber kul(.)turprogramm

017 spricht vielleicht nicht JEden an.

018 [viele wollen dann ja auf ihrer abschlussFAHRT(-)

019 ET: [hm_hm,

$020 \mathrm{KI}$ : eher was mit der KLASse machen,

$021=$ [und nicht unbedingt [kultTUR-

022 ET: [hm_hm,

023

024

025

026

027

028

029

030

031

032

033

034

035

036

037

038

039

040 ET: also EIN tag (-) hin und r'für [hin und rückfahrt,

$041 \mathrm{KI}: \quad[\mathrm{ja}$;

$((. .)$.

hm_hm,

ja-

${ }^{\circ} \mathrm{h}$ ich hätte so auCH (-) die den gedanken dazu,

${ }^{\circ} \mathrm{h}(-)$ dass ähm wenn man DREI tage hat,

$(--)$ [von der ANreise her;

KI : $\quad[j a$;

ET: also je nach[dem wieviel GELD (-) eh [man be KOMMT,

$\mathrm{KI}: \quad[j a-$

ET: entweder (.) FLUGzeug,

(ob) das dann überhaupt MÖGlich ist, =weil DREI tage (-) mit_dem BUS da irgendwie hin,

KI : ja;

ET: eh das ist ja ne straPAze [oder,

KI : $\quad$ [ja und HAMburg wäre dann

042 ET: ${ }^{\circ} \mathrm{h}$ ja was haben wir NOCH;

$043=$ =vier TAge bungalowpark,

044 also center PARK oder (-) so irgendwas;

$045 \mathrm{KI}:$ mit FREIzeitmöglichkeiten;

046 ET: ja;

047 ja so sowas wie (---) wie du schon kennst; 
048

049

050

051

052

053

054

056

057

058

059

060

061

062

063

064

065

066

067

068

069

070

071

072

073

074

075

076

077

078

079

080

081

082

083

084

085

086

KI : ja;

[das ist (-) AUCH nicht schlecht,

ET: [also so so BUNgalow, bungalowpark, (3.0) je nachdem ob man in verschiedenen HÄUsern ist, dann SO was weiß ich; immer (1.0) acht oder zehn leute in so_einem BUNGALOW;

KI: ja JE nachDEM ne, [das (.) is ja imMER,

ET : $\quad[j a-$ (5.0)

KT: also in[die engere AUSwahl würde ich schon mal entweder HAMburg oder BUNGALOW [park-

ET : $\quad[$ hm_hm, ja;

${ }^{\circ} \mathrm{h}$ also in MEIne engere auswahl auch.

KI: aber dann,

ET: [also EINS und-

KI : [beim bungalowpark ich also man kann halt SHOPPEN und SCHWIMmen, aber das könnte man HIER ja auch;

[also hier kann man ja AUCH als klasse schwimmen gehen;

ET: [hm_hm,

KI : $\quad$ [also das finde ich JETZT

ET: $\quad\left[\mathrm{hm} \_\mathrm{hm}\right.$, MEIN favorit wäre eins und vier .

KI : [jaha;

ET: [im moment so vom geFÜHL; und bei DIR?

[was wäre dein, KI : $\quad$ [AUch;

ET: ein auch bei dir [ein-

KI : $\quad$ [wobei ich glaub ich sogar NOCH mehr zu [EINS tendiere.

ET: $\quad$ [EINS, okay; 
087

KI: weil (--) hamburg ist halt ne große STADT,

$088 \quad$ [es haben viele noch nicht geSEhen,

089 ET: [hm_hm,

090 hm_hm,

Die Aushandlung in EZP 3 beginnt damit, dass das Elternteil sich die Aufgabenstellung schrittweise vor Augen führt und sich sein Verständnis vom Kind bestätigen lässt (Z. 7). Damit attribuiert die Mutter dem Kind lokal eine höhere Kompetenz im Verstehen der Aufgabe. Es ist diesmal das Kind, das gleich gesprächsorganisatorisch die Optionen thematisiert (Z. 8, 11) und bewertet (Z. 9), was die Mutter wiederum nur bestätigt (Z. 10). Es ist ebenfalls das Kind, das einen Dissens bzw. ein Problem etabliert (Heller 2012), (Z. 16: das ist natürlich schön aber) und sofort selbst einen möglichen Einwand gegen diese Option ausführt (Z. 16-20). Die Mutter bestätigt (Z. 22) und fügt einen weiteren Einwand gegen diese Option hinzu (Z. 2637), der dann vom Kind als unterstützende Begründung für eine weitere Option genutzt wird (Z. 38-41).

Das Kind steuert den Entscheidungsdiskurs sehr aktiv, indem es die nunmehr (längere Auslassung im Transkript) von beiden favorisierten Alternativoptionen zusammenfasst (Z. 60), was das Elternteil ratifiziert (Z. 65). Als das Elternteil wiederum offenbar in den Entscheidungsdiskurs einsteigt, unterbricht das Kind, indem es selbstständig eine Begründung für eine Option formuliert, diese dann aber selbst wieder entkräftet (Z. 68-72). Im Folgenden konkurrieren beide Beteiligte in der Kontextualisierung des Diskurses (Z. 75f.): Das Elternteil bleibt auf der Entscheidungsebene, das Kind argumentiert.

Wie auch die vorherigen Aushandlungen des 2. Beispiels ist die Aushandlung zum EZP 3 grundsätzlich von einem Konsens zwischen Elternteil und Kind geprägt. Das Elternteil akzeptiert Begründungen und leistet keine Unterstützung im Argumentationsdiskurs, sondern fordert lediglich Entscheidungen und Resümees vom Kind ein. Weil das Elternteil dazu neigt, häufig nur Kriterien für die Entscheidung zu nennen, ohne jedoch die Gültigkeit des Kriteriums für einzelne Optionen zu präzisieren, sowie Optionen unbegründet aufgreift und wieder fallenlässt (Z. 27 f., 34f., 40, 76f., 82), hat das Kind - im Vergleich zum Fall 1001 - weniger konkrete Anschlussmöglichkeiten, um zu argumentieren.

Das Kind folgt über die ersten beiden EZPe eher den organisatorischen Gesprächssteuerungen des Elternteils und löst sich davon erst im EZP 3 vermehrt durch Eigeninitiative beim Produzieren von Entscheidungen und Begründungen, indem es z. B. unaufgefordert Optionen gegenüberstellt. Hinsichtlich des Argumentationsdiskurses ist bemerkenswert, dass die Neuntklässlerin - wie erwähnt - fast gegen die Mutter, jedenfalls nicht mit ihrer 
Unterstützung, argumentative gegenüber rein entscheidungsorganisierenden Verfahren umsetzt.

Wie nach drei Jahren erwartbar, ist entsprechend auch bei diesem Kind eine Steigerung der Argumentationskompetenz erkennbar. Im Unterschied zum Kind in Beispiel (1) lassen sich diese Zuwächse jedoch nicht mit den interaktiven Erfahrungen, die das Kind im Verlauf seiner Interaktionsgeschichte mit dem Elternteil gemacht hat, in Verbindung bringen: Dieses Kind konnte keine elterlichen Modelle für die Begründungspflicht und für begründende Verfahren nutzen. Seine Begründungen bleiben entsprechend rein lokal, werden also nicht global ausgebaut oder strukturell komplexer (z. B. in Form von Abwägungen). Der Gesprächsraum, der dem Kind im Rahmen von Raumlassen und Akzeptieren (im Unterschied zu Übergehen und Selberlösen) gewährt wird, lässt seine Kompetenzsteigerung in Form einer höheren und andersartigen Beteiligung des Kindes sichtbar werden; es gibt aber keinen Hinweis darauf, dass das Kind die interaktiven Erfahrungen im Dialog mit seiner Mutter systematisch als Ressource für die Kompetenzsteigerung nutzen konnte.

Wie sich die drei Interaktionsmuster im gesamten Korpus hinsichtlich der längsschnittlichen Veränderungen verhalten, wird im nächsten Abschnitt beschrieben.

\subsection{Beobachtungen und Häufigkeitsverteilungen im gesamten Längsschnitt}

Die Analysen aller 33 Eltern-Kind-Dyaden im Längsschnittvergleich zeigen, dass bei allen Kindern - unabhängig vom rekonstruierten Interaktionsmuster die selbstständig produzierten Anteile in den Aushandlungen über die Zeit ansteigen. Diese Beobachtung entspricht der Annahme, dass der Grad an Selbstständigkeit des Kindes bzw. die allgemeine Diskurskompetenz im Zuge der kognitiven, sozialen und sprachlichen Entwicklung zwischen der 6. und der 9. Jahrgangsstufe generell zunimmt. Indizien dafür sind sowohl die vergleichsweise längere Gesprächsdauer ${ }^{10}$ als auch eine Verschiebung in der Bewältigung diskursrelevanter Aufgaben vom Elternteil auf das Kind. Diese vollzieht sich sowohl in Entscheidungs- als auch in Argumentationssequenzen.

10 Die allgemeine Gesprächsdauer erhöht sich beim EZP 3 (9. Klasse) gegenüber dem EZP 1 (6. Klasse) um durchschnittlich $20 \%$. 
Wo also bei Fordern und Unterstützen typischerweise in EZP 1 noch das Elternteil in Form von lokalen Zugzwängen ,Schritt für Schritt‘ Entscheidungen und Begründungen des Kindes evozieren oder modellhaft vorlegen musste, wird diese fordernde Unterstützungsleistung aufgrund der Eigeninitiative des Kindes im Verlauf der EZPe - wie in Beispiel (1) - überflüssig.

Nur vereinzelt findet eine solche Verschiebung vom Elternteil auf das Kind über den Längsschnitt auch in der globalen Organisation des gesamten Gesprächs statt. Die eröffnende globale Organisation des Aufgabendiskurses sowie das Abschließen in Form der Feststellung, dass eine Einigung herbeigeführt und damit die Aufgabe erfüllt wurde, verbleiben typischerweise über alle EZPe beim Elternteil, unabhängig von der Entwicklung des Kindes beim Begründen. Es gibt zwar Fälle, in denen das Kind durchaus auch beim globalen Gesprächsmanagement den Willen zur Partizipation andeutet, jedoch werden diese Übernahmen i.d.R. vom Elternteil nicht geduldet. Deutlich wird dies beispielsweise bei dem Phänomen des doppelten Abschließens, in dem das Elternteil den kindseitig selbstständig vertexteten Abschluss des Argumentationsdiskurses nicht einfach ratifiziert, sondern als Abschluss des Aufgabendiskurses seinerseits wiederholt. Diese Beobachtungen belegen, dass und wie die Dyaden sich in der Tat hör- und sichtbar auch der von uns gestellten Aufgabe zuwenden (s. Quasthoff in diesem Band) und nicht nur entscheiden und argumentieren. Sie zeigen darüber hinaus, wie sich auch bei Neuntklässlern i. Allg. noch die asymmetrische Rollenbeziehung ElternKind manifestiert.

Die kindlichen Eigenleistungen bei der Produktion von Entscheidungsund Argumentationssequenzen unter Einbezug der Interaktionsmuster können überblicksartig in Häufigkeitsverteilungen gebündelt werden, die im Folgenden anhand von Tabellen dargestellt werden.

Tabelle 1 listet alle 33 Eltern-Kind-Dyaden der Intensivstichprobe nach rekonstruierten Interaktionsmustern auf und bezieht sie auf den Entscheidungsdiskurs. In der Spalte Anstieg über den Längsschnitt erscheint die Zahl der Eltern-Kind-Dyaden, bei denen die Analysen eine deutliche Verschiebung in den Entscheidungssequenzen vom Elternteil auf das Kind über den Längsschnitt zeigen. In der rechten Spalte ist die Anzahl jener Eltern-Kind-Dyaden vermerkt, bei denen sich keine Erhöhung der selbstständigen Beiträge des Kindes finden lassen (vgl. Quasthoff et al. 2015):

Besonders bei Raumlassen und Akzeptieren wie auch bei Fordern und Unterstützen produzieren die Kinder in EZP 1 noch häufig fremdinitiierte Entscheidungen als Folge elterlicher Zugzwänge, wie in den beiden Beispielen in 5.2 und 5.1 oben gezeigt wurde, während sie in EZP 3 ihre Präferenzen ini- 
Tab. 1: Anstieg vs. Konstanz von selbstständig initiierten Entscheidungssequenzen bezogen auf die Interaktionsmuster der Familien.

\begin{tabular}{lrr}
\hline \multirow{2}{*}{$\begin{array}{l}\text { Familiales } \\
\text { Interaktionsmuster }\end{array}$} & $\begin{array}{r}\text { Erhöhung/Konstanz von kindseitig selbstständig initiierten } \\
\text { Entscheidungssequenzen über den Längsschnitt }\end{array}$ \\
\cline { 2 - 4 } & $\begin{array}{r}\text { Anstieg über den } \\
\text { Längsschnitt }\end{array}$ & $\begin{array}{r}\text { Konstanz über den } \\
\text { Längsschnitt }\end{array}$ \\
\cline { 2 - 4 } Fordern und Unterstützen & 13 & 2 \\
\hline Raumlassen und Akzeptieren & 6 & $2^{11}$ \\
\hline Übernehmen und Selberlösen & 6 & 3 \\
\hline insgesamt & 25 & 7 \\
\hline
\end{tabular}

tiativ ins Gespräch einbringen. Auch bei Übernehmen \& Selberlösen steigt die Tendenz zu selbstständigen Entscheidungen über den Längsschnitt überraschenderweise in 6 Fällen an. Bei genauerer Analyse stellt sich jedoch heraus, dass die Kinder in diesen Fällen i.d.R. nicht - wie bei Fordern und Unterstützen - im Rahmen der kooperativen Organisation des Entscheidungsdiskurses stärker partizipieren. Vielmehr lehnen die Kinder typischerweise häufiger elterliche Lösungen einfach ab oder insistieren auf einer vom Elternteil verworfenen Option (,ich find aber XY doch besser“). Es geht also eher um Widerstand gegen die elterliche Dominanz als um eine aktivere Rolle in der Organisation des Entscheidungsdiskurses.

Die Tab. 2 zeigt die Entwicklung kindseitiger Übernahmen beim Argumentieren. Sie zeigt einen hohen Anstieg selbstinitiierter Argumentationssequenzen beim Muster Fordern und Unterstützen, ganz im Gegensatz zu Eltern-Kind-Dyaden des Musters Übergehen und Selberlösen. Diese bleiben über den Längsschnitt überwiegend konstant: Nur bei zwei von neun Fällen zeigt sich eine erhöhte

11 Das Muster Raumlassen und Akzeptieren ist hier in einem Fall nicht berücksichtigt: Zu Fall: K5 = 617 kann im Hinblick auf eine längsschnittliche Untersuchung mit Fokus auf kindliche Kompetenzzunahme keine zuverlässigen Aussage gemacht werden, da das Kind in EZP 1 und 2 äußerst unkooperatives Verhalten zeigt sowie sämtliche Revisionsaufgaben nicht aufgabenkonform bearbeitet wurden. 
Tab. 2: Anstieg vs. Konstanz von selbstständig initiierten Argumentationssequenzen bezogen auf die Interaktionsmuster der Familien.

\begin{tabular}{lcr}
\hline $\begin{array}{l}\text { Familiales } \\
\text { Interaktionsmuster }\end{array}$ & $\begin{array}{r}\text { Erhöhung/Konstanz von kindseitig selbstständig initiierten } \\
\text { Argumentationssequenzen über den Längsschnitt }\end{array}$ \\
\cline { 2 - 4 } & $\begin{array}{r}\text { Anstieg über den } \\
\text { Längsschnitt }\end{array}$ & $\begin{array}{r}\text { Konstanz über den } \\
\text { Längsschnitt }\end{array}$ \\
\cline { 2 - 4 } Fordern und Unterstützen & 11 & $4^{12}$ \\
\hline Raumlassen und Akzeptieren & $4^{13}$ & 4 \\
\hline Übernehmen und Selberlösen & 2 & 7 \\
\hline
\end{tabular}

Produktion selbstständiger Argumentationssequenzen. ${ }^{14}$ Bemerkenswert ist, dass Kinder, die über den gesamten Längsschnitt Übernehmen und Selberlösen ,ausgesetzt‘ waren, zuallermeist auf der Entscheidungsebene verbleiben, obwohl ihnen insbesondere in EZP 3 durchaus genug Raum für Argumentationssequenzen bleibt.

Das Muster Raumlassen und Akzeptieren zeigt keinen eindeutigen Trend. Während die Hälfte der Kinder durchaus einen z. T. deutlichen Anstieg selbstständig produzierter Argumentationssequenzen aufweist, stagniert wiederum die Entwicklung bei anderen. Man kann vermuten, dass bereits das Angebot von Gesprächsraum, in dem das Kind grundsätzlich die Möglichkeit zur Produktion eigener Anteile hat, auch ohne Unterstützungsleistung von Seiten des Elternteils besonders bei argumentativ starken Kindern zu einer Übernahme von diskursrelevanten Gesprächsanteilen führt.

Insgesamt zeigen die Längsschnittanalysen, dass vor allem geforderte und unterstützte Kinder im Verlauf der EZPe zu selbstständigem Argumentieren neigen. Sie erkennen die kontextuelle Notwendigkeit für (elaborierte) Begründungen von Entscheidungspräferenzen häufiger von sich aus. Ebenso begründen diese Kinder häufiger die Einwände gegen die Entscheidungen des Eltern-

$12 \mathrm{Zu}$ den Fällen der rechten Spalte (Konstanz über den Längsschnitt) zählt auch ein Kind, das über den gesamten Längsschnitt, also bereits ab EZP 1, ungewöhnlich hohe Selbstständigkeit zeigt und sich dann nicht mehr steigert.

13 siehe Fußnote 11.

14 Diese Fälle konnten bereits ab EZP 1 nicht eindeutig einem Interaktionsmuster zugewiesen werden. So wurden bei jenen Fällen von den Analysierenden bei der Zuordnung zu Übergehen und Selberlösen zusätzlich die Bemerkung Mit Ansätzen zum Fordern ergänzt. Beide Fälle wechseln im EZP 3 zum Muster Raumlassen und Akzeptieren. 
teils. Die rekonstruktiven Einblicke lassen dabei auf Grund der Passung zwischen der Art der elterlichen Steuerungen und der Veränderungen in den kindlichen Beiträgen durchaus die Annahme zu, dass diese Kompetenzsteigerung wesentlich auf den Einfluss der interaktiven Erfahrungen zurückzuführen ist, die die Kinder als Erwerbsressource nutzen (vgl. Quasthoff, Wild et al. in diesem Band zu den quantitativ-statistischen Bezügen).

\section{Fordern und Unterstützen als Erwerbsressource in unterschiedlichen kommunikativen Familienkulturen als Chance oder Risiko für die schulrelevante Sprachentwicklung}

Die in 5.1 beschriebenen interaktiven Mechanismen und die in 5.3 berichteten Verteilungen legen eindeutig nahe, dass die Interaktionspraktik Fordern und Unterstützen den familialen Erwerbskontext kennzeichnet, der die stärkste Erwerbsunterstützung für argumentative Diskursfähigkeiten bietet. Selbst die vier Fälle dieses Musters, in denen der Grad der Selbstständigkeit stagniert, lassen sich erklären: Wie oben bereits beschrieben, gibt es hierunter einen Fall, bei denen bereits im EZP 1 eine überdurchschnittlich hohe Eigenbeteiligung des Kindes auffällt, die sich über den Längsschnitt hinweg dann nicht weiter fortentwickelt. Hier ist das Kind also hochkompetent und das entscheidende Entwicklungsfenster liegt offensichtlich bereits vor dem von uns erfassten Zeitraum. Bei zwei weiteren Fällen verweigern die Kinder zumeist, trotz Unterstützungsleistung des Elternteils, die Mitarbeit und verweigern sich besonders bei EZP 3. Die elterliche Unterstützungsleistung bleibt über den Längsschnitt konstant.

Unabhängig von der methodisch nicht zentralen Frage, ob sich im Einzelfall in der rekonstruktiven Analyse Erklärungen für nicht theoriekonforme Erwerbsverläufe finden lassen, kann abschließend die zentrale Rolle des Finetuning festgehalten werden. Nur in dem Maße, in dem die dialogischen Zugzwänge das Kind passgenau im Rahmen seiner Möglichkeiten fordern und unterstützen, also in Vygotskis (1978) „Zone der nächsten Entwicklung“ steuern, können sie vom Kind als Erwerbsressource genutzt werden (Heller \& Krah 2015). Wie die Diskussion im Abschnitt 5.1 gezeigt hat, lässt sich dieses intuitiv adaptive elterliche Verhalten erst im Längsschnitt überprüfen. 
Da die Intensivstichprobe in der FUnDuS-Studie in ihrer Zusammensetzung (Abschnitt 3.1 oben; Quasthoff in diesem Band) den immer wieder statistisch bestätigten Zusammenhang zwischen Sprachkompetenz und sozioökonomischem Status aus heuristischen Gründen aufzubrechen versuchte, konnten wir zur Verteilung der Muster feststellen, dass Fordern und Unterstützen zwar vornehmlich in Familien mit hohem, aber auch in solchen mit niedrigem Sozialstatus vorkommt. Eher sozial benachteiligte Familien praktizierten - relativ gleich verteilt - alle drei Muster (Quasthoff et al. 2015). Daraus ist zu schließen, dass Fordern und Unterstützen als kommunikative Praktik nicht an eine privilegierte Familienkultur gekoppelt zu sein scheint. Dies ist die Voraussetzung für die Hoffnung, dass ein entsprechendes Elterntraining (Kluger in diesem Band) die Erwerbsbedingungen für Kinder auch und gerade in sozial benachteiligten Familien verbessern kann.

\section{Literatur}

Arendt, Birte (2019): Argumentieren mit Peers: Erwerbsverläufe und -muster bei Kindergartenkindern. Tübingen: Stauffenburg.

Arendt, Birte, Vivien Heller \& Antje Krah (2015): Kinder argumentieren. Interaktive Erwerbskontexte und -mechanismen: Zur Einführung. Mitteilungen des Deutschen Germanistenverbandes 62 (1), 1-4.

Becker-Mrotzek, Michael, Karen Schramm, Eike Thürmann \& Helmut Johannes Vollmer, (Hrsg.) (2013): Sprache im Fach. Sprachlichkeit und fachliches Lernen. Münster: Waxmann.

Bergmann, Jörg \& Thomas Luckmann (1995): Reconstructive genres of everyday communication. In Uta Quasthoff, (Hrsg.), Aspects of oral communication, 289-304. Berlin, Boston: De Gruyter.

Bergmann, Jörg \& Uta Quasthoff (2010): Interaktive Verfahren der Wissensgenerierung: Methodische Problemfelder. In Ulrich Dausendschön-Gay, Christine Domke \& Sören Ohlhus, (Hrsg.), Wissen in (Inter)Aktion. Verfahren der Wissensgenerierung in unterschiedlichen Praxisfeldern, 21-34. Berlin: De Gruyter.

Bruner, Jerome (1978): The role of dialogue in language acquisition. In Anne Sinclair, Robert Jarvella \& Willem Levelt, (Hrsg.), The Child's Conception of Language, 241-256. Berlin: Springer.

Burleson, Brant, Jesse Delia \& James Applegate (1995): The Socialization of Person-Centered Communication. Parents' Contributions to Their Children's Social-Cognitive and Communication Skills. In Mary Anne Fitzpatrick \& Anita Vangelisti, (Hrsg.), Explaining Family Interactions, 34-76. London, New Dehli: Sage.

Ehlich, Konrad (2012): Basisqualifikationen in der schulischen Entfaltung - ein Beispiel (Diskursivität). In Konrad Ehlich, Renate Valtin \& Beate Lütke (Hrsg.), Expertise „Erfolgreiche Sprachförderung unter Berücksichtigung der besonderen Situation Berlins“, 65-79. Berlin: Senatsverwaltung für Bildung, Jugend und Wissenschaft.

Ehlich, Konrad \& Jochen Rehbein (1986): Muster und Institution: Untersuchungen zur schulischen Kommunikation. Tübingen: Narr. 
Erath, Kirstin \& Susanne Prediger (2014): Was wird zum Erklären gelernt? Konstitution eines Lerngegenstands in der Klasseninteraktion. In Jürgen Roth \& Judith Ames (Hrsg.), Beiträge zum Mathematikunterricht 2014, 345-348. Münster: WTM.

Feilke, Helmuth (2013): Bildungssprache und Schulsprache - am Beispiel literalargumentativer Kompetenzen. In Michael Becker-Mrotzek, Karen Schramm, Eike Thürmann \& Helmut Johannes Vollmer (Hrsg.), Sprache im Fach. Sprachlichkeit und fachliches Lernen, 113-130. Münster: Waxmann.

Garnica, Olga (1977): Some prosodic and paralinguistic features of speech to young children. In Catherine E. Snow \& Charles A. Ferguson (Hrsg.), Talking to children: Language input and acquisition, 63-88. Cambridge etc.: Cambridge University Press.

Goetz, Peggy (2010): The development of verbal justifications in the conversations of preschool children and adults. First Language 30, 403-420.

Grundler, Elke (2011): Kompetent argumentieren. Ein gesprächsanalytisch fundiertes Modell. Tübingen: Stauffenburg.

Hausendorf, Heiko (1995): Deixis and Orality. Explaining Games in Face to Face Interaction. In Uta Quasthoff (Hrsg.), Aspects of Oral Communication, 181-197. Berlin, Boston: De Gruyter.

Hausendorf, Heiko \& Uta Quasthoff (1996): Sprachentwicklung und Interaktion: Eine linguistische Studie zum Erwerb von Diskursfähigkeiten bei Kindern. Wiesbaden: Westdeutscher Verlag. Neu aufgelegt bei: Verlag für Gesprächsforschung: http://www.verlag-gespraechsforschung.de/2005/quasthoff.htm

Hausendorf, Heiko \& Uta Quasthoff (2005): Konversations-/Diskursanalyse: (Sprach-) Entwicklung durch Interaktion. In Günter Mey (Hrsg.), Qualitative Forschung in der Entwicklungspsychologie, 585-618. Köln: Kölner Studien.

Heller, Vivien (2012): Kommunikative Erfahrungen von Kindern in Familie und Unterricht. Passungen und Divergenzen. Tübingen: Stauffenburg.

Heller, Vivien \& Antje Krah (2015): Wie Eltern und Kinder argumentieren. Interaktionsmuster und ihre Erwerbsförderlichkeit im längsschnittlichen Vergleich. Mitteilungen des Deutschen Germanistenverbandes 62 (1), 5-20.

Heller, Vivien \& Miriam Morek (2015): Unterrichtsgespräche als Erwerbskontext: Kommunikative Gelegenheiten für bildungssprachliche Praktiken erkenn und nutzen. Leseforum.ch, 2015 (3), Themenheft Literalität im Schnittfeld von Familie, Frühbereich und Schule, 1-23.

Hollmann, Jelena, Elke Wild, Uta Quasthoff, Antje Krah \& Nantje Otterpohl (2012): Der Erwerb sprachlicher Kompetenzen im Elternhaus - Erste Befunde zur Güte eines Fragebogens zur Erfassung des Anregungsgehalts von Familienkonversationen. Unterrichtswissenschaft 40, 47-63.

Kern, Friederike (2011): Der Erwerb kommunikativer Praktiken und Formen - Am Beispiel des Erzählens und Erklärens. In Habscheid, Stephan (Hrsg.), Textsorten, Handlungsmuster, Oberflächen. Linguistische Typologien der Kommunikation, 231-253. Berlin: De Gruyter.

Klann-Delius, Gisela, Klaus Blaschko, Heiko Hausendorf et al. (1985): Untersuchungen zur Entwicklung von Diskursfähigkeit am Beispiel von Spielerklärungen. Linguistische Arbeiten und Berichte 21, 1-436.

Krah, Antje (2017): Gespräche in der Familie als unterrichtsähnliche Interaktion. (Normative) Rahmungen argumentativer Entscheidungsdiskurse. In Martin Luginbühl \& Stefan Hauser (Hrsg.), Gesprächskompetenz in schulischer Interaktion - normative Ansprüche, 189-215. Bern: hep. 
Krah, Antje, Uta Quasthoff, Vivien Heller, Elke Wild, Jelena Hollmann \& Nantje Otterpohl (2013): Die Rolle der Familie beim Erwerb komplexer sprachlicher Fähigkeiten in der Sekundarstufe I. In Angelika Redder \& Sabine Weinert (Hrsg.), Sprachförderung und Sprachdiagnostik. Perspektiven aus Psychologie, Sprachwissenschaft und empirischer Bildungsforschung, 68-88. Münster: Waxmann.

Krelle, Michael (2014): Mündliches Argumentieren in leistungsorientierter Perspektive: Eine empirische Analyse von Unterrichtsdiskussionen in der neunten Jahrgangsstufe.

Baltmannsweiler: Schneider Verlag.

Miller, Max (1980): Zur Ontogenese moralischer Argumentation. Zeitschrift für Literaturwissenschaft und Linguistik 10 (38/39), 58-108.

Morek, Miriam (2012): Kinder erklären. Interaktionen in Familie und Unterricht im Vergleich. Tübingen: Stauffenburg.

Morek, Miriam, Vivien Heller \& Uta Quasthoff. (2017): Erklären und Argumentieren: Modellierungen und empirische Befunde zu Strukturen und Varianzen. In Iris Meißner \& Eva Wyss (Hrsg.), Begründen, Erklären, Argumentieren, 11-46. Tübingen: Stauffenburg.

Nelson, Katherine (1977): Facilitating children's syntax acquisition. Developmental Psychology 13, 101-107.

Quasthoff, Uta (2009): Entwicklung der mündlichen Kommunikationskompetenz. In Michael Becker-Mrotzek, (Hrsg.), Unterrichtskommunikation und Gesprächsdidaktik. Teilband Mündlichkeit in der Handbuchreihe Deutschunterricht in Theorie und Praxis, 84-100. Baltmannsweiler: Schneider Verlag.

Quasthoff, Uta (2011): Diskurs- und Textfähigkeiten: Kulturelle Ressourcen ihres Erwerbs. In Ludger Hoffmann, Kerstin Leimbrink \& Uta Quasthoff (Hrsg.), Die Matrix der menschlichen Entwicklung, 210-251. Berlin: De Gruyter.

Quasthoff, Uta (2012): Aktual- und mikrogenetische Zugänge zur Ontogenese: Inspirationen der Konversationsanalyse zur Verbindung von sprachlichen Praktiken und dem Erwerb sprachlicher Kompetenzen. In Ruth Ayaß \& Christian Meyer (Hrsg.), Sozialität in Slow Motion: Theoretische und empirische Perspektiven, 217-244. Wiesbaden: Springer VS.

Quasthoff, Uta \& Nitza Katz-Bernstein (2006): Diskursfähigkeiten. In Manfred Grohnfeldt (Hrsg.), Lexikon der Sprachtherapie, 72-75. Stuttgart: Kohlhammer.

Quasthoff Uta \& Friederike Kern (2007): Familiale Interaktionsmuster und kindliche Diskursfähigkeit: Mögliche Auswirkungen interaktiver Stile auf diskursive Praktiken und Kompetenzen bei Schulkindern. In Hausendorf, Heiko (Hrsg.), Gespräch als Prozess. Linguistische Aspekte der Zeitlichkeit verbaler Interaktion, 277-306. Tübingen: Narr.

Quasthoff, Uta, Lilian Fried, Nitza Katz-Bernstein, Anke Lengning, Anja Schröder \& Juliane Stude (2011): (Vor)Schulkinder erzählen im Gespräch: Kompetenzunterschiede systematisch erkennen und fördern. Das Dortmunder Beobachtungsinstrument zur Interaktions- und Narrationsentwicklung (DO-BINE) und der Dortmunder Förderansatz (DO-FINE). Baltmannsweiler: Schneider Verlag.

Quasthoff Uta \& Antje Krah (2012): Die familiale Kommunikation zwischen den Generationen als Ressource für Diskurserwerb. In Eva Neuland (Hrsg.), Sprache der Generationen, 115-132. Mannheim: Duden.

Quasthoff, Uta \& Elke Wild (2014): Learning in context from an interdisciplinary perspective. Learning, Culture and Social Interaction 2014 (3), 69-76.

Quasthoff, Uta, Elke Wild, Madeleine Domenech, Jelena Hollmann, Christian Kluger, Antje Krah, Nantje Otterpohl \& Johannes Wibbing (2015): Mündliches und schriftliches 
Argumentieren in der Sekundarstufe I: Können Eltern ihre Kinder beim Erwerb dieser Schlüsselkompetenz unterstützen? In Angelika Redder, Johannes Naumann \& Rosemarie Tracy (Hrsg.), Forschungsinitiative Sprachdiagnostik und Sprachförderung - Ergebnisse, 207-233. Münster: Waxmann.

Quasthoff, Uta \& Madeleine Domenech (2016): Theoriegeleitete Entwicklung und Überprüfung eines Verfahrens zur Erfassung von Textqualität (TexQu) am Beispiel argumentativer Briefe in der Sekundarstufe I. Didaktik Deutsch 41, 19-41.

Quasthoff, Uta, Vivien Heller \& Miriam Morek (2017): On the sequential organization and genre-orientation of discourse units in interaction: An analytic framework. Discourse Studies 19 (1), 84-110.

Quasthoff, Uta, Friederike Kern, Sören Ohlhus \& Juliane Stude (2019): Diskurse und Texte von Kindern: Praktiken - Fähigkeiten - Ressourcen: Erwerb. Tübingen: Stauffenburg. http://dx.doi.org/10.17877/DE290R-20360

Rezat, Sara (2011): Schriftliches Argumentieren. Zur Ontogenese konzessiver Argumentationskompetenz. Didaktik Deutsch 17(31), 50-67.

Rowe, Meredith L. (2008): Child-directed speech: relation to socioeconomic status, knowledge of child development and child vocabulary skill. Journal of Child Language 35, 185-205.

Sacks, Harvey (1995): Lectures on conversation. Volumes I \& II. Edited by Jefferson J. Oxford: Blackwell.

Schegloff, Emanuel A. (1972): Sequencing in Conversational Openings. In John Gumperz \& Dell Hymes (Hrsg.), Directions in sociolinguistics. The Ethnography of Communication, 346-380. New York: Holt, Rinehart and Winston.

Selting, Margret, Peter Auer, Dagmar Barth-Weingarten, Jörg Bergmann, Pia Bergmann, Karin Birkner, Elizabeth Couper-Kuhlen, Arnulf Deppermann, Peter Gilles, Susanne Günthner, Martin Hartung, Friederike Kern, Christine Mertzlufft, Christian Meyer, Miriam Morek, Frank Oberzaucher, Jörg Peters, Uta Quasthoff, Wilfried Schütte, Anja Stukenbrock, Susanne Uhmann (2009): Gesprächsanalytisches Transkriptionssystem 2 (GAT 2). Gesprächsforschung - Online-Zeitschrift zur verbalen Interaktion 10, 353-402. http://www.gespraechsforschung-ozs.de/heft2009/px-gat2.pdf.

Snow, Catherine (1995): Issues in the Study of Input: Finetuning, Universality, Individual and Developmental Differences, and Necessary Causes. In Paul Fletcher \& Brian MacWhinney (Hrsg.), The Handbook of Child Language, 180-193. Oxford: Blackwell.

Snow, Catherine \& Charles Ferguson (Hrsg.) (1977): Talking to children: language input and acquisition. Cambridge: Cambridge University Press.

Spiegel, Carmen (2006): Unterricht als Interaktion. Gesprächsanalytische Studien zum kommunikativen Spannungsfeld zwischen Lehrern, Schülern und Institution. Radolfzell: Verlag für Gesprächsforschung.

Spreckels, Janet (2008): „Ham die dir’s schon erklärt?“ Worterklärungen im schulischen und außerschulischen Kontext. Muttersprache 118 (2), 121-145.

Stanat, Petra \& Aileen Edele (2014): Migration und soziale Ungleichheit. In Heinz Reinders, Helmut Ditton, Cornelia Gräsel \& Burkhard Gniewosz, (Hrsg.), Empirische Bildungsforschung: Gegenstandsbereiche, (2. Aufl.), 181-192. Opladen: VS Verlag.

Steinig, Wolfgang (2016) Sprache, Bildung und soziale Herkunft. In Jörg Kilian, Birgit Brouër \& Dina Lüttenberg (Hrsg.), Handbuch Sprache in der Bildung, 68-98. Berlin: De Gruyter.

Tillmann, Klaus-Jürgen (2001): Basislager für Leistung und Schulerfolg: Wie die kulturelle Mitgift der Eltern wirkt. In Gerold Becker, Imbke Behnken, Marianne Horstkemper, Uwe Jopt \& Klaus-Jürgen Tillmann (Hrsg.), Schülerheft Familie, 124-127. Velber: Friedrich. 
Völzing, Paul-Ludwig (1981): Kinder argumentieren. Die Ontogenese argumentativer Fähigkeiten. Paderborn: Schöningh.

Vygotsky, Lev (1978): Mind in society. The development of higher psychological processes. Cambridge, MA: Harvard University Press.

Wald, Benji (1978) Zur Einheitlichkeit und Einleitung von Diskurseinheiten. In Uta Quasthoff (Hrsg.), Sprachstruktur - Sozialstruktur, 128-149. Königstein: Scriptor.

Walper, Sabine \& Elke Wild (2014): Lernumwelten in der Familie. In Tina Seidel \& Andreas Krapp (Hrsg.), Pädagogische Psychologie, 359-385. Weinheim: Belz.

Willenberg, Heiner, Michael Krelle \& Steffen Gailberger (2007): Argumentation. In Bärbel Beck \& Eckhard Klieme (Hrsg.): Sprachliche Kompetenzen. Konzepte und Messungen. DESIStudie, 118-129. Weinheim: Beltz. 